\title{
GNSS Signal Authentication via Power and Distortion Monitoring
}

\author{
Kyle D. Wesson, Member, IEEE, Jason N. Gross, Member, IEEE, Todd E. Humphreys, Member, IEEE, and \\ Brian L. Evans, Fellow, IEEE
}

\begin{abstract}
We propose a simple low-cost technique that enables civil Global Positioning System (GPS) receivers and other civil global navigation satellite system (GNSS) receivers to reliably detect carry-off spoofing and jamming. The technique, which we call the Power-Distortion detector, classifies received signals as interference-free, multipath-afflicted, spoofed, or jammed according to observations of received power and correlation function distortion. It does not depend on external hardware or a network connection and can be readily implemented on many receivers via a firmware update. Crucially, the detector can with high probability distinguish low-power spoofing from ordinary multipath. In testing against over 25 high-quality empirical data sets yielding over 900,000 separate detection tests, the detector correctly alarms on all malicious spoofing or jamming attacks while maintaining a $<0.6 \%$ single-channel false alarm rate.
\end{abstract}

Index Terms-satellite navigation systems, Global Positioning System, Global Navigation Satellite Systems, navigation security, GNSS spoofing, GNSS jamming

\section{INTRODUCTION}

$\mathbf{G}$ NSS receivers are tremendously popular in navigation and timing applications due to their accuracy, low cost, and global operation. Low cost can be credited to the fact that civil GNSS signals are defined in freely-available, openaccess standards [1], [2], which makes receiver development straightforward. But an open-access standard, together with civil GNSS signals' near-perfect predictability, invites forgery: receivers can fall victim to spoofing attacks in which counterfeit GNSS signals fool the receiver into reporting a hazardously misleading position or time [3]. The vulnerability of civil GNSS receivers to spoofing is a serious risk for GNSS-dependent critical infrastructure and safety-of-life applications [4], [5].

GNSS authentication techniques can be broadly categorized into three groups: (1) cryptographic techniques that exploit unpredictable but verifiable signal modulation in the GNSS

Manuscript submitted on DD MMM 2017

K. Wesson was supported in part by DoD, Air Force Office of Scientific Research, National Defense Science and Engineering Graduate Fellowship 32 CFR $\$ 168 \mathrm{a}$, and The University of Texas at Austin Microelectronics and Computer Development Fellowship. J. Gross was supported in part by a West Virginia University Big XII Faculty Fellowship. T. Humphreys was supported in part by the National Science Foundation under Grant No. 1454474 (CAREER).

K. Wesson, B. Evans are with the Department of Electrical and Computer Engineering, and T. Humphreys with the Department of Aerospace Engineering and Engineering Mechanics, at The University of Texas at Austin, Austin, TX 78712 USA (e-mail: kyle.wesson@utexas.edu, todd.humphreys@mail.utexas.edu, bevans@ece.utexas.edu). J. Gross is with the Department of Mechanical and Aerospace Engineering at West Virginia University (e-mail: jason.gross@mail.wvu.edu). spreading code or navigation data, (2) geometric techniques that exploit the angle-of-arrival diversity of authentic GNSS signals, and (3) GNSS signal processing techniques that do not fall into categories (1) or (2). A comprehensive survey of GNSS authentication techniques is offered in [6].

Cryptographic signal authentication is effective [6]-[8], but, despite recent interest and engagement by U.S. and European satellite navigation agencies [9], [10], no open civil GNSS signals yet incorporate cryptographic modulation. Moreover, it has become clear that financial and technical hurdles will impede development and implementation of such modulation for years to come. It is possible to leverage the existing encryption of military GNSS signals for civil signal authentication [11], but this technique requires the protected receiver to be connected to a data network, an undesirable dependency that prevents stand-alone operation.

Authentication techniques that exploit GNSS signals' geometric diversity can also be highly effective. These include angle-of-arrival discrimination techniques based on multiple antennas [12]-[15], or a single antenna experiencing oscillatory motion [16], or a single antenna with multiple feeds [17]. The drawback of these approaches is their reliance on multiple antennas, antenna motion, or an assumption that interference signals arrive from below the antenna's horizon. Likewise, methods that require coupling with inertial sensors [18], [19] or vision sensors may prove impractical in applications with cost, size, weight, or power constraints.

Practical near-term GNSS signal authentication techniques are those that do not require changes to GNSS signals-inspace, are receiver-autonomous, low-cost, require no additional hardware, and can be implemented via a software or firmware update. Recognizing the value of techniques that fall into this category, previous work has proposed monitoring the total received power via the Automatic Gain Control (AGC) setpoint [20], and monitoring autocorrelation profile distortion [21]-[23]. But when acting separately these techniques are unreliable for signal authentication. A received power monitor that ignores correlation distortion may not detect a low-power spoofer. Moreover, because a power-monitoringonly technique does not distinguish between spoofing and jamming, its alarm rate can become intolerable in urban areas where so-called personal privacy devices (PPDs, small GNSS jammers) [24] are common. For their part, the distortionmonitoring approaches in [21]-[23] ignore total received power, and thus can be fooled by a spoofer transmitting with a significant power advantage over the authentic signals, which, by action of the AGC, forces the authentic signals under the 
noise floor, leaving a distortion-free correlation function [8].

A GNSS authentication technique is needed that is both practical in the sense described above and reliable at detecting spoofing. To this end, we propose to combine the elemental tests mentioned earlier, namely, detection of anomalous received power and detection of correlation profile distortion, in a GNSS signal authentication technique we call the PowerDistortion detector, or PD detector for short. The key insight behind our approach is this: The practically-unavoidable interaction between authentic and spoofed GNSS signals during the initial stages of a tracking-points-carry-off spoofing attack makes such spoofing evident, with high probability, in received power or signal distortion or both. If a carry-off-type spoofer transmits at a low signal power, the attack will either be ineffective or will cause significant correlation function distortion as the similarly-sized spoofing and authentic signals interact. On the other hand, if a spoofer transmits at a high signal power, the correlation function may be distortion-free but the receiver's total received power will be anomalously high. Our proposed technique traps a would-be spoofer between simultaneous measurements of received power and correlation function distortion. Provided the spoofer is unable to block or otherwise null the authentic GNSS signals impinging on the receiver's antenna (a difficult task if the receiver enjoys a physical security perimeter [6]), the combination of these measurements within a Bayesian detection framework poses a formidable defense against carry-off-type spoofing.

This paper, a significant extension of our work in [25], makes three main contributions. First, it introduces a novel technique for detecting GNSS jamming and carry-off-type spoofing and rigorously develops the measurement models and probability distributions required to characterize the detection statistic. Second, it presents a Monte-Carlo-type method for determining the Bayes-optimal decision rule and offers detailed consideration of the requisite cost function. Third, it presents a thorough evaluation of the proposed technique against three realistic data sets: (1) the Texas Spoofing Test Battery [26]-[28], a public set of GPS spoofing recordings; (2) the RNL Multipath and Interference Recordings [29], [30], a public set of deep urban GNSS recordings with significant multipath; and (3) a set of recordings of GNSS signals subject to jamming.

\section{Signal Models}

\section{A. Pre-Correlation Model}

Consider the following generic representation of an authentic GNSS signal exiting a receiver's radio frequency (RF) front-end downconversion chain. For notational compactness, the signal is expressed by its complex baseband representation,

$$
r_{\mathrm{A}}(t)=\sqrt{P_{\mathrm{A}}} D\left(t-\tau_{\mathrm{A}}\right) C_{r}\left(t-\tau_{\mathrm{A}}\right) \exp \left(j \theta_{\mathrm{A}}\right)
$$

where $t$ is time in seconds, $P_{\mathrm{A}}$ is the received power of the authentic signal in Watts, $D(t)$ is the \pm 1 -valued navigation data modulation, $C_{r}(t)$ is the \pm 1 -valued pseudorandom spreading (ranging) code, $\tau_{\mathrm{A}}$ is the code phase in seconds, and $\exp \left(j \theta_{\mathrm{A}}\right)$ is the carrier with phase $\theta_{\mathrm{A}}$ in radians. $P_{\mathrm{A}}, \tau_{\mathrm{A}}$, and $\theta_{\mathrm{A}}$ are assumed to vary with time; their time dependency is suppressed for notational simplicity. Without loss of generality for the purposes of this paper, the navigation data modulation $D(t)$ can be ignored; hence, hereafter we assume $D(t)=1$.

Let $r_{\mathrm{I}}(t)$ represent a single complex-valued interference signal that is structurally identical to $r_{\mathrm{A}}(t)$. This could be a multipath, spoofing, or jamming signal. If multipath, $r_{\mathrm{I}}(t)$ represents the strongest reflection at time $t$, whose effect on received power and correlation function distortion is a good proxy for that of the aggregate multipath. If jamming, $r_{\mathrm{I}}(t)$ represents structured-signal jamming, an especially potent form of jamming similar to spoofing except there is no expected correlation between the jamming signal's code or carrier phase and those of $r_{\mathrm{A}}(t)$ [31]. The interference signal is modeled as

$$
r_{\mathrm{I}}(t)=\sqrt{\eta P_{\mathrm{A}}} C_{r}\left(t-\tau_{\mathrm{I}}\right) \exp \left(j \theta_{\mathrm{I}}\right)
$$

where $\eta=P_{\mathrm{I}} / P_{\mathrm{A}}$ is the interference signal's power advantage relative to the authentic signal, and $\tau_{\mathrm{I}}$ and $\theta_{\mathrm{I}}$ are the interference signal's code and carrier phase, respectively.

To complete the received signal model, let

$$
r_{\mathrm{N}}(t)=N(t)+M(t)
$$

be a white zero-mean complex-valued Gaussian process that models the sum of thermal noise $N(t)$, with constant spectral density $N_{0}$, and multi-access interference $M(t)$, with variable spectral density $M_{0}$. The two noise components are assumed to be independent so that $r_{\mathrm{N}}(t)$ has density $N_{0}+M_{0} . M(t)$ accounts for the noise contribution from other legitimate GNSS signals besides the desired signal (collectively called multiaccess signals), and any interference accompanying these. With the addition of $r_{\mathrm{N}}(t)$, the full received signal-plusinterference-and-noise model is given by

$$
r(t)=r_{\mathrm{A}}(t)+r_{\mathrm{I}}(t)+r_{\mathrm{N}}(t)
$$

As shown in Fig. 11, an AGC circuit is assumed to apply a scaling factor $\beta(t)$ to $r(t)$ so that the power in the scaled signal $\beta(t) r(t)$ remains constant. Subsequent to AGC scaling, the signal is quantized and encoded; for simplicity, these operations are ignored in Fig. 1 and in the remainder of this paper, as their effects on the this paper's detection processing are negligible.

At the core of GNSS signal processing is correlation of $\beta(t) r(t)$ with a local replica

$$
\ell(t, \tau)=C_{\ell}(t-\hat{\tau}-\tau) \exp (j \hat{\theta})
$$

where $\tau$ is an arbitrary code phase lag in seconds and $C_{\ell}(t)$ is the local code replica, which, ignoring the effects of bandlimiting on the received signal, is often made equal to $C_{r}(t)$. The goal of a receiver's code and carrier tracking loops is to drive the estimates $\hat{\tau}$ and $\hat{\theta}$ to match $\tau_{\mathrm{A}}$ and $\theta_{\mathrm{A}}$ as accurately as possible. In practice, however, $\hat{\tau}$ and $\hat{\theta}$ track the code and carrier phase of the composite signal $r(t)$, not just those of $r_{\mathrm{A}}(t)$.

\section{B. Post-Correlation Model}

Correlation and accumulation over an interval $T$ ending at time $t_{k}=k T, k \in\{1,2, \ldots\}$ produce the complex-valued 


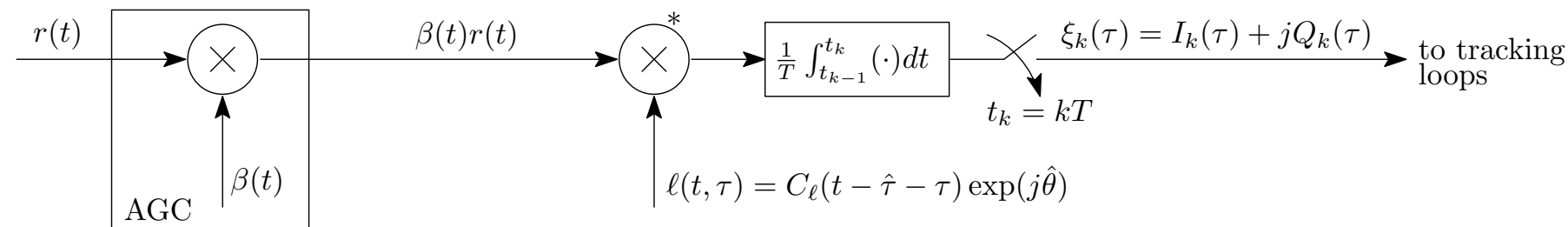

Fig. 1: Block diagram of the standard AGC, correlation, and accumulation operations in a GNSS receiver. The product of the AGC-scaled incoming signal $\beta(t) r(t)$ and the conjugate of the local replica $\ell(t, \tau)$ is accumulated over $T$ seconds to produce the discrete complex-valued accumulation product $\xi_{k}(\tau)$. For notational convenience, the accumulation product has been scaled by $1 / T$.

accumulation product $\xi_{k}$, which, when viewed as a function of the arbitrary lag $\tau$ introduced by the local replica $\ell(t, \tau)$, is called the receiver's correlation function for signal $r_{\mathrm{A}}(t)$ at time $t_{k}$, and is modeled as [32]

$$
\xi_{k}(\tau)=\beta_{k}\left[\xi_{\mathrm{A} k}(\tau)+\xi_{\mathrm{I} k}(\tau)+\xi_{\mathrm{N} k}(\tau)\right]
$$

where $\beta_{k}$ is the average value of $\beta(t)$ over the $k$ th accumulation interval, and $\xi_{\mathrm{A} k}(\tau), \xi_{\mathrm{I} k}(\tau), \xi_{\mathrm{N} k}(\tau)$ are the complex correlation function components corresponding to the authentic signal, the interference signal, and thermal noise, respectively. Fig. 2 illustrates such a correlation function.

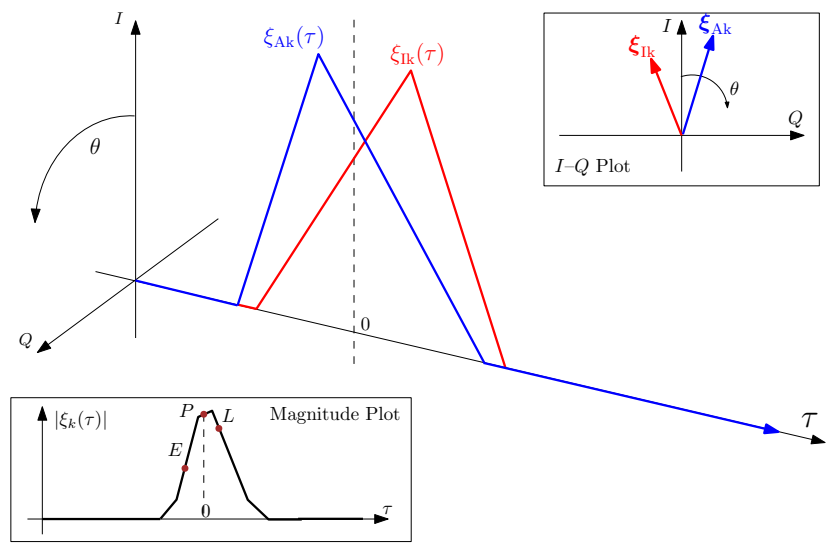

Fig. 2: Components of the correlation function $\xi_{k}(\tau)$ for an example scenario with a triangular-shaped $R(\tau)$ and a strong spoofing or multipath interference component $\xi_{\mathrm{I} k}(\tau)$. For visual clarity, the thermal noise component $\xi_{\mathrm{N} k}(\tau)$ is not shown. The upper-right inset shows the phase angle of the authentic and interference components relative to the in-phase $I$ and quadrature $Q$ components of the local replica $\ell(t, \tau)$. The lower-left inset shows the magnitude of the composite correlation function $\xi_{k}(\tau)$, which is clearly distorted by the interference component. $E, P$, and $L$ mark the early, prompt, and late correlation taps, respectively. The local replica's code phase estimate $\hat{\tau}$ attempts to track the code phase of the composite signal $r(t)$ by equalizing $E$ and $L$. The prompt tap $P$ is located at $\tau=0$.

The function $R(\tau)=E\left[C_{r}(t) C_{\ell}(t-\tau)\right]$, often called the autocorrelation function of $C_{r}(t)$ even though, strictly speaking, $C_{\ell}(t)$ may be slightly different from $C_{r}(t)$, approximates the interaction between $C_{r}(t)$ and $C_{\ell}(t)$ over the correlation and accumulation operations:

$$
R(\tau) \approx \frac{1}{T} \int_{t_{k-1}}^{t_{k}} C_{r}(t) C_{\ell}(t-\tau) d t
$$

The correlation components $\xi_{\mathrm{A} k}(\tau)$ and $\xi_{\mathrm{I} k}(\tau)$ can be modeled in terms of $R(\tau)$ as

$$
\begin{aligned}
\xi_{\mathrm{A} k}(\tau) & =\sqrt{P_{\mathrm{A} k}} R\left(-\Delta \tau_{\mathrm{A} k}+\tau\right) \exp \left(j \Delta \theta_{\mathrm{A} k}\right) \\
\xi_{\mathrm{I} k}(\tau) & =\sqrt{\eta_{k} P_{\mathrm{A} k}} R\left(-\Delta \tau_{\mathrm{I} k}+\tau\right) \exp \left(j \Delta \theta_{\mathrm{I} k}\right)
\end{aligned}
$$

where $P_{\mathrm{A} k}$ and $\eta_{k}$ are the average values of $P_{\mathrm{A}}$ and $\eta$ over the accumulation interval, and $\Delta \tau_{\mathrm{A} k}$ is the average value of the difference $\tau_{\mathrm{A}}-\hat{\tau}$ over the accumulation interval, with similar definitions for $\Delta \tau_{\mathrm{I} k}, \Delta \theta_{\mathrm{A} k}$, and $\Delta \theta_{\mathrm{I} k}$.

The thermal noise component of the correlation function, $\xi_{\mathrm{N} k}(\tau)$, is modeled as having independent in-phase (real) and quadrature (imaginary) components, each modeled as a zeromean Gaussian white discrete-time process:

$$
E\left[\mathbb{R}\left\{\xi_{\mathrm{N} k}(\rho)\right\} \mathbb{I}\left\{\xi_{\mathrm{N} j}(\nu)\right\}\right]=0 \quad \forall \rho, \nu, k \neq j
$$

The chip interval of the spreading code $C_{r}(t)$, denoted $\tau_{c}$, ranges from 0.01 to $1 \mu \mathrm{s}$ in modern GNSS signals. Due to the pseudorandom nature of $C_{r}(t)$, only samples of $\xi_{\mathrm{N} k}(\tau)$ within $2 \tau_{c}$ of each other are correlated [33]:

$E\left[\xi_{\mathrm{N} k}(\rho) \xi_{\mathrm{N} k}^{*}(\nu)\right]= \begin{cases}2 \sigma_{\mathrm{N}}^{2}\left(1-|\rho-\nu| / \tau_{c}\right) & |\rho-\nu| \leq 2 \tau_{c} \\ 0 & |\rho-\nu|>2 \tau_{c}\end{cases}$

Here, ${ }^{*}$ denotes the complex conjugate and $\sigma_{\mathrm{N}}^{2}$ is the variance of the in-phase and quadrature components of $\xi_{\mathrm{N} k}(\tau)$, which is related to the spectral density of the white noise process $r_{\mathrm{N}}(t)$ by $\sigma_{\mathrm{N}}^{2}=\left(N_{0}+M_{0}\right) / 2 T$.

\section{Hypothesis TESTING FRAMEWORK}

We adopt a Bayesian $\mathrm{M}$-ary hypothesis testing framework [34, Ch. 2] for distinguishing between hypotheses $H_{i}, i \in \mathcal{I}$, where $\mathcal{I}=\{0,1,2,3\}$. The null hypothesis $H_{0}$ corresponds to the interference-free case, and $H_{i}, i=1,2,3$ correspond respectively to multipath, spoofing, and jamming.

The foregoing signal models reveal three parameters relevant to choosing between hypotheses, namely, the interference power advantage $\eta$, and the interference-to-authentic code and carrier offsets $\Delta \tau \triangleq \tau_{\mathrm{I}}-\tau_{\mathrm{A}}$ and $\Delta \theta \triangleq \theta_{\mathrm{I}}-\theta_{\mathrm{A}}$. Let these be combined into a single vector $\theta=[\eta, \Delta \tau, \Delta \theta]^{T}$ assumed to lie in the parameter space $\Lambda$, itself divided into disjoint parameter 
sets $\Lambda_{i} \subset \Lambda, i \in \mathcal{I}$, each associated with its corresponding hypothesis $H_{i}$. Thus, deciding that $\theta \in \Lambda_{i}$ is equivalent to choosing hypothesis $H_{i}$. Note that, because $\theta$ can take on a range of values, the hypothesis testing problem is composite.

In a Bayesian formulation of the composite hypothesis testing problem, the parameter vector $\theta$ is viewed as a random quantity, $\Theta$, having density $w(\theta)$, with $\pi_{i} \triangleq P\left(\Theta \in \Lambda_{i}\right)$ being the prior probability that $\Theta$ falls in $\Lambda_{i}$. We denote by $w_{i}(\theta)$ the conditional density of $\Theta$ given that $\Theta \in \Lambda_{i}$; it follows that

$$
w_{i}(\theta)= \begin{cases}0 & \theta \notin \Lambda_{i} \\ w(\theta) / \pi_{i} & \theta \in \Lambda_{i}\end{cases}
$$

We propose to decide between the four hypotheses based on two types of observation at each $t_{k}$, namely, the received power measurement $P_{k}$ and the symmetric difference measurement $D_{k}$, both detailed in a later section. The observation vector $z_{k}=\left[D_{k}, P_{k}\right]^{T}$, which resides in the observation set $\Gamma$, is modeled as a random variable $Z_{k}$ with conditional density $p\left(z_{k} \mid \theta\right) . H_{i}$ can be defined as the hypothesis that $Z_{k}$ is distributed as $p\left(z_{k} \mid \Theta \in \Lambda_{i}\right), i \in \mathcal{I}$.

A decision rule $\delta\left(z_{k}\right)$ is a partition of $\Gamma$ into disjoint decision regions $\Gamma_{i}, i \in \mathcal{I}$, such that $H_{i}$ is chosen when $z_{k} \in \Gamma_{i}$ :

$$
\delta\left(z_{k}\right)= \begin{cases}0 & \text { if } z_{k} \in \Gamma_{0} \\ 1 & \text { if } z_{k} \in \Gamma_{1} \\ 2 & \text { if } z_{k} \in \Gamma_{2} \\ 3 & \text { if } z_{k} \in \Gamma_{3}\end{cases}
$$

Let $C[i, \theta]$ be the cost of choosing $H_{i}$ when $\theta \in \Lambda$ is the actual parameter vector. Note that this function is sensitive to a particular value of $\theta$, which makes it more general than one that simply assigns a unform cost for choosing $H_{i}$ when $\theta \in \Lambda_{j}$. A later section will introduce various embodiments of $C[i, \theta]$.

An optimum rule selects the least costly hypothesis, on average, given the observation $z_{k}$. More precisely, if we define the conditional risk, or the average cost for $\Theta=\theta$, as

$$
R_{\theta}(\delta) \triangleq E_{\theta}\left\{C\left[\delta\left(Z_{k}\right), \theta\right]\right\}, \quad \theta \in \Lambda
$$

where $E_{\theta}$ denotes expectation assuming $Z_{k} \sim p\left(z_{k} \mid \theta\right)$, and if we define average, or Bayes, risk as

$$
r(\delta)=E\left[R_{\Theta}(\delta)\right]
$$

where the expectation is now taken over the random quantity $\Theta$, then the optimum rule $\delta$ is the one whose decision regions $\Gamma_{i}, i \in \mathcal{I}$, minimize $r(\delta)$.

To find the minimizing $\delta$, each parameter set $\Lambda_{i}$ and conditional distribution $w_{i}(\theta)$ must be defined for $i \in \mathcal{I}$. These could be approximated from an extensive campaign of empirical multipath, spoofing, and jamming data collection and analysis. But any empirical characterization runs the risk of biasing the $\Lambda_{i}$ and $w_{i}(\theta)$ toward the particular dataset used. This is merely a restatement of Hume's problem of induction: metaphorically speaking, the empirical dataset may only contain white swans [35]. To avoid this pitfall of inference, which, in a security context, could be a serious vulnerability, the following definitions of $\Lambda_{i}$ and $w_{i}(\theta)$ are informed not only by observation but also by the physical characteristics and limitations of signals under the various hypotheses.

\section{$H_{0}:$ No interference}

In what follows, we denote the marginal distributions of $w_{i}(\theta)$ by $w_{\eta i}(x), w_{\Delta \tau i}(x)$, and $w_{\Delta \theta i}(x), i \in \mathcal{I}$. Under the null hypothesis $(i=0)$, which corresponds to the interferencefree case, we have

$$
\Lambda_{0}=\{\theta \in \Lambda \mid \eta=0\}
$$

It follows that $w_{\eta 0}(x)$ is equivalent to the Dirac delta function. The marginal distributions $w_{\Delta \tau 0}(x)$, and $w_{\Delta \theta 0}(x)$ can be defined arbitrarily, since for $\eta=0$ they have no effect on $r(\delta)$.

\section{$H_{1}:$ Multipath}

In the case of multipath, $\eta$ and $\Delta \tau$ can be bounded as follows:

$$
\Lambda_{1}=\left\{\theta \in \Lambda \mid 0<\eta<\eta_{1}, 0<\Delta \tau<\Delta \tau_{1}\right\}
$$

The bounds $\eta_{1}$ and $\Delta \tau_{1}$ are informed by the physics of signal reception and signal processing, as follows. Whenever the authentic signal is unobstructed, it arrives with greater power than any echo, whose additional path length and interaction with reflection surfaces invariably attenuate its power [36]. Moreover, an echo whose delay is more than double the spreading code chip interval (e.g., more than $0.2 \mu$ s for $\tau_{c}=0.1 \mu \mathrm{s}$ ) causes no correlation function distortion [33], and, due to the additional path loss, is at least $3 \mathrm{~dB}$ weaker than an unobstructed authentic signal [37]. Thus, its effect on $r(\delta)$ is negligible. It follows that $\eta_{1}=1$ and $\Delta \tau_{1}=2 \tau_{c}$ upper-bound the multipath parameter set for an unobstructed authentic signal.

On the other hand, the severe shadowing experienced by mobile receivers can cause $\eta>1$, especially in urban environments where surrounding buildings simultaneously attenuate and reflect authentic signals [37]. In these situations, there is no practical upper limit on $\eta$ because the direct-path authentic signal may be attenuated by $50 \mathrm{~dB}$ or more. It is not possible to reliably distinguish multipath from low-power spoofing in such circumstances.

One can avoid this difficulty by applying this paper's detection test only when the authentic signal $r_{\mathrm{A}}(t)$ is received without severe shadowing, in which case the probability that $\eta>1$ under $H_{1}$ again becomes negligible. In particular, the multipath model developed below assumes that $r_{\mathrm{A}}(t)$ is attenuated less than $6 \mathrm{~dB}$ by shadowing. In practice, one simply excludes cases in which the received power $P_{k}$ is not unusually high yet the measured carrier-to-noise ratio $C / N_{0}$ drops by more than $6 \mathrm{~dB}$ from its modeled value for an unobstructed authentic signal.

An analysis of GNSS multipath was carried out to validate the bounds $\eta_{1}=1$ and $\Delta \tau_{1}=2 \tau_{c}$ and to characterize $w_{1}(\theta)$. The analysis was based on the Land Mobile Satellite Channel Model (LMSCM) [38], itself based on extensive experimentation with a wideband airborne transmitter at GNSS frequencies in urban and suburban environments [37], [38]. The LMSCM generates power, delay, and carrier phase for both line-of-sight and echo signals using deterministic models for attenuation, 
diffraction, and delay that respond to stochastically-generated obstacles and reflectors in the simulation environment.

In keeping with the philosophy of robust detection, multipath scenarios were sought whose distribution $w_{1}(\theta)$ was most similar to the distribution for spoofing, $w_{2}(\theta)$. From the standpoint of distinguishing multipath from spoofing, this is the worst-case $w_{1}(\theta)$. As a practical matter, the worst-case $w_{1}(\theta)$ has a high proportion of large $\eta$ values and a wide range of $\Delta \theta$. Not surprisingly, urban LMSCM scenarios with low-elevation satellite signals yielded the worst-case $w_{1}(\theta)$.

For flexibility of detector design, the simulation-derived $w_{1}(\theta)$ was parameterized in terms of satellite elevation angle $\alpha_{e}$. Several 1-minute LMSCM simulations with randomlycreated urban environments and various satellite azimuth angles were run for $\alpha_{e} \in[20,80]$ degrees. At each simulation epoch, the maximum-amplitude echo was identified and its relative power, delay, and phase with respect to the line-ofsight signal were taken as sampled $\eta, \Delta \tau$, and $\Delta \theta$ values from which $w_{1}(\theta)$ could be approximated. Epochs whose line-ofsight signal was attenuated by more than $6 \mathrm{~dB}$ were excluded, resulting in exclusion of up to $90 \%$ of epochs for heavilyshadowed scenarios at $\alpha_{e}=20$, but less than $1 \%$ of epochs at $\alpha_{e}=80$. At each $\alpha_{e}$, the scenario producing the worst-case $w_{1}(\theta)$ was selected.

A statistical analysis of the sampled $\eta, \Delta \tau$, and $\Delta \theta$ values revealed that the relative phase $\Delta \theta$ was uniformly distributed on $[0,2 \pi)$ and independent of $\eta, \Delta \tau$, which is unsurprising given the wavelength-scale sensitivity of $\Delta \theta$ to path length. The parameters $\eta$ and $\Delta \tau$ were found to be significantly correlated, with a linear correlation coefficient of approximately $\rho=-0.23$, implying that more distant reflectors tend to produce weaker echos. Consistent with [36], the marginal distribution $w_{\eta 1}(x)$ was found to be log-normal, with a mean of $-21 \mathrm{~dB}$ and a standard deviation of $5 \mathrm{~dB}$, both insensitive to $\alpha_{e}$. Thus, excluding cases of heavy line-of-sight signal shadowing, multipath causes $\eta>1$ with negligible probability, which indicates that $\eta_{1}=1$ is an unproblematic bound. The marginal distribution $w_{\Delta \tau 1}(x)$ was found to be exponential,

$$
w_{\Delta \tau 1}(x)=\mu^{-1} e^{-x / \mu}, \quad x \geq 0
$$

with $\mu$ a quadratic function of $\alpha_{e}$,

$$
\mu=0.012 \alpha_{e}^{2}-2.4 \alpha_{e}+134
$$

where $\alpha_{e}$ is expressed in degrees and $\Delta \tau$ in nanoseconds. Thus, $\Delta \tau$ values are widely spread at low $\alpha_{e}$ but tightly clustered near zero at high $\alpha_{e}$, consistent with the empirical distributions in [37].

\section{$\mathrm{H}_{2}$ : Spoofing}

Under the spoofing hypothesis, we take $\eta_{1}=1$ as a lower bound for $\eta$ and assume $|\Delta \tau|$ is upper-bounded by $\Delta \tau_{1}=2 \tau_{c}$ :

$$
\Lambda_{2}=\left\{\theta \in \Lambda\left|\eta_{1} \leq \eta, 0<\right| \Delta \tau \mid<\Delta \tau_{1}\right\}
$$

These bounds make sense because the spoofing signal must be at least as powerful as the authentic signal for reliable spoofing, and because spoofing whose $|\Delta \tau|$ value exceeds $\Delta \tau_{1}=2 \tau_{c}$ is no longer classified as carry-off-type spoofing, since the interference is uncorrelated with the authentic signal. Instead, for $|\Delta \tau| \geq \Delta \tau_{1}$, the attack is classified as jamming.

For maximum stealth, the distribution $w_{2}(\theta)$ should be as close as possible to $w_{1}(\theta)$ while respecting the bounds $\eta_{1}$ and $\Delta \tau_{1}$ and allowing a wide range of $\Delta \tau$ for code pull-off. Thus, $w_{\eta 2}(x)$ is modeled as log-normal with mean of $1 \mathrm{~dB}$ and standard deviation a fraction of $\mathrm{a} \mathrm{dB}, w_{\Delta \tau 2}(x)$ as exponential with parameter $\mu=120$ (mimicking low-elevation multipath), and $w_{\Delta \theta 2}(x)$ as uniform on $[0,2 \pi)$.

\section{$H_{3}$ : Jamming}

Because it is uncorrelated with the authentic signal, jamming is taken to have $|\Delta \tau| \geq \Delta \tau_{1}=2 \tau_{c}$. Its power advantage is assumed to be at least $\eta_{1}=1$, as weaker jamming is both harmless and so common as to be unremarkable. Thus, the jamming parameter set is

$$
\Lambda_{3}=\left\{\theta \in \Lambda\left|\eta_{1} \leq \eta, \Delta \tau_{1} \leq\right| \Delta \tau \mid\right\}
$$

The marginal distribution $w_{\eta 3}(x)$ is taken to be Rician. By adjusting the Rician distance and scale parameters $\nu$ and $\sigma$, one can model high- or low-power jamming within a wide or narrow range. The marginal distributions $w_{\Delta \tau 3}(x)$ and $w_{\triangle \theta 3}(x)$ can be modeled arbitrarily, as they have no effect on $r(\delta)$ since $|\Delta \tau| \geq \Delta \tau_{1}$ eliminates correlation with the authentic signal.

\section{Measurement Models}

This section develops models for the two observations that constitute the interference detection statistic, namely, the received power measurement and the symmetric difference measurement.

\section{A. Received Power Measurement}

The total received power measured by a GNSS receiver in an RF band of interest, denoted $P_{k}$ for measurement time $t_{k}$, is a simple and effective indicator of interference [20], [39]. Even subtle spoofing attacks with power advantage $\eta$ near unity can cause an increase in $P_{k}$ that is distinguishable from random variations, including so-called nulling attacks that attempt to suppress the authentic signals [31]. The catch is that routine events such as solar radio bursts and the near passage of a so-called personal privacy device can also cause $P_{k}$ to rise above nominal levels [31]. Thus, to prevent an anomalouspower monitor from issuing alarms intolerably often, the alarm threshold may need to be raised to a point where the monitor is no longer sensitive to subtle spoofing attacks. This is why power monitoring is best coupled with other forms of interference monitoring.

Suppose $M_{s}+1$ authentic signals modeled as (1) are received, each with its associated interference signal, modeled as (2). Let $r_{\mathrm{A} i}(t)$ and $r_{\mathrm{I} i}(t)$ represent the $i$ th authentic and interference signals. The combined signal exiting the RF front end is then

$$
r_{\mathrm{C}}(t)=\sum_{i=0}^{M_{s}}\left[r_{\mathrm{A} i}(t)+r_{\mathrm{I} i}(t)\right]+N(t)
$$




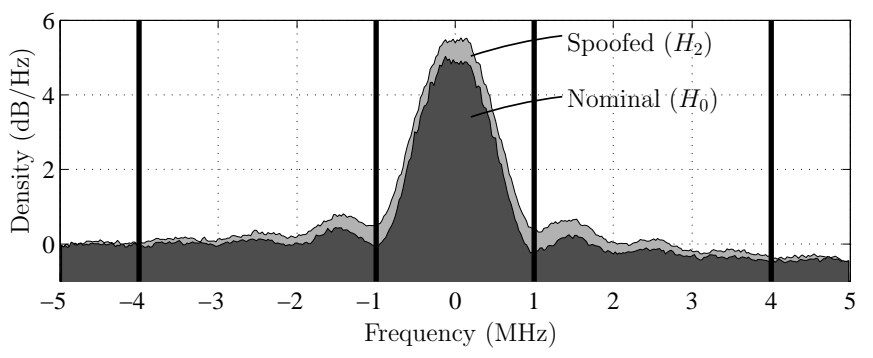

Fig. 3: Normalized power spectral density about the GPS L1 C/A center frequency $(1575.42 \mathrm{MHz})$ for a static receiver platform during nominal conditions in a clean RF environment (dark gray), and during a spoofing attack (light gray). The two spectra are superimposed so that, of the spoofing attack spectrum, only the excess power beyond nominal is visible. The inner two vertical lines represent the 2-MHz bandwidth about $\mathrm{L} 1$, the outer two the $8-\mathrm{MHz}$ bandwidth. The spoofing attack spectrum applies at $t_{k}=130$ seconds into scenario 7 of the Texas Spoofing Test Battery (TEXBAT tb 7), described in [28].

where $N(t)$ is the same thermal noise component as in (3).

Receivers with sufficient dynamic range in the discrete samples produced from $r_{\mathrm{C}}(t)$ do not require an AGC and so can compute received power directly by averaging the squared modulus of these samples. Let $W_{P}$ be the bandwidth over which $P_{k}$ is to be measured. If $W_{P}$ is narrower than the receiver front-end's native bandwidth, then $r_{\mathrm{C}}(t)$ must be filtered to isolate the desired spectral interval. Fig. 3 shows 2and 8-MHz bands for $P_{k}$, with Fig. 4 showing corresponding power time histories. In a receiver whose front end is equipped with an AGC, $P_{k}$ is measured indirectly through the AGC setpoint [20]. In this case $W_{P}$ is equivalent to the front-end noise-equivalent bandwith.

Let $\tilde{r}_{\mathrm{C}}(t)$ represent the (optionally) filtered version of $r_{\mathrm{C}}(t)$ from which $P_{k}$ is to be measured. $P_{k}$ is calculated as the average power in $\tilde{r}_{\mathrm{C}}(t)$ over the interval $t_{k-1}$ to $t_{k}$ :

$$
P_{k}(\mathrm{dBW}) \triangleq 10 \log _{10}\left(\frac{1}{T} \int_{t_{k-1}}^{t_{k}}\left|\tilde{r}_{\mathrm{C}}(t)\right|^{2} d t\right)
$$

Let $p_{P_{k}}(x \mid \theta)$ be the conditional density of $P_{k}$. Assuming $W_{P}$ is wide enough that $\tilde{r}_{\mathrm{C}}(t)$ retains the significant power in $r_{\mathrm{A} i}(t)$ and $r_{\mathrm{I} i}(t)$, then $p_{P_{k}}(x \mid \theta)$ can be modeled as Gaussian, i.e., $p_{P_{k}}(x \mid \theta)=\mathcal{N}\left(x ; \bar{P}_{k}, \sigma_{P}^{2}\right)$, where $\bar{P}_{k}$ and $\sigma_{P}$ are expressed in $\mathrm{dBW}$. The mean, $\bar{P}_{k}$, revealed by carrying out the multiplications inherent in $\left|\tilde{r}_{\mathrm{C}}(t)\right|^{2}=\tilde{r}_{\mathrm{C}}(t) \tilde{r}_{\mathrm{C}}^{*}(t)$, and by noting that $N(t)$ is zero-mean and independent of $r_{\mathrm{A} i}(t)$ and $r_{\mathrm{I} i}(t)$, and that, due orthogonality of spreading codes, $E\left[r_{\mathrm{A} i}(t) r_{\mathrm{A} i}^{*}(t)\right]=E\left[r_{\mathrm{I} i}(t) r_{\mathrm{I} l}^{*}(t)\right]=0$ for all $i \neq l$, is $\bar{P}_{k}=10 \log _{10}\left(\bar{P}_{\mathrm{L}}\right)$, where

$$
\begin{aligned}
\bar{P}_{\mathrm{L}}= & \sum_{i=0}^{M_{s}}\left[\left(1+\eta_{i}\right) P_{\mathrm{A} i}+2 \sqrt{\eta_{i}} P_{\mathrm{A} i} \cos \left(\Delta \theta_{i}\right) R\left(\Delta \tau_{i}\right)\right] \\
& +N_{0} W_{P}
\end{aligned}
$$

and the subscript $i$ associates the corresponding quantity with the $i$ th signal. The first term in the summation is the non-
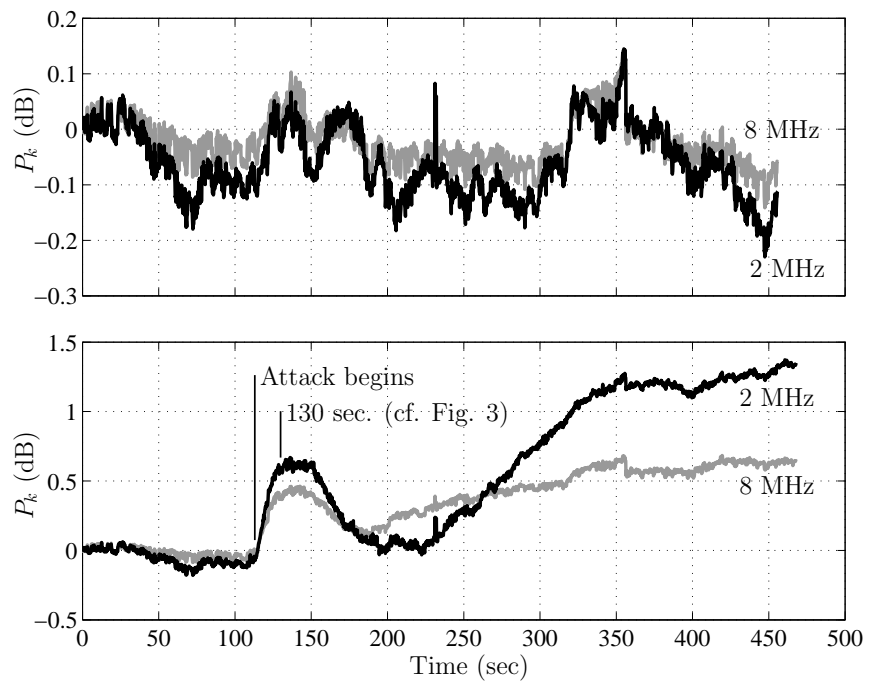

Fig. 4: Time histories of measured received power $P_{k}$ for the nominal scenario (top) and spoofing scenario (bottom) whose spectra are shown in Fig. 3. Black traces correspond to the $2-\mathrm{MHz}$ bandwith and gray traces to the $8-\mathrm{MHz}$ bandwidth centered at L1. The averaging interval for each measurement is $200 \mathrm{~ms}$. $P_{k}$ has been normalized to $0 \mathrm{~dB}$ at $t_{k}=0$. The 130second mark noted in the bottom panel is the instant at which the spoofing attack spectrum in Fig. 3 applies. At this point in the attack, $\eta_{i} \approx 4, \Delta \theta_{i} \approx \pi$, and $\Delta \tau_{i} \approx 0$ for $i=0, \ldots, M_{s}$.

coherent sum of power in $r_{\mathrm{A} i}$ and $r_{\mathrm{I} i}$; the second term is the power contributed by coherent interaction between $r_{\mathrm{A} i}$ and $r_{\mathrm{I} i}$. If $\eta_{i}=0$, or if the interference is orthogonal to the authentic signal (e.g., $\Delta \theta_{i}= \pm \pi / 2$ ), or if $\Delta \tau_{i}$ is large enough that $R\left(\Delta \tau_{i}\right)=0$, then this second term vanishes.

The deviation $\sigma_{P}$ about $\bar{P}_{k}$ accounts for unpredictable but natural variations in $P_{k}$ such as measurement error due to the finite duration over which $P_{k}$ is estimated. From the top plot of Fig. 4, one can see that for the receiver under test, $\sigma_{P}$ is at approximately $0.1 \mathrm{~dB}$ and $0.05 \mathrm{~dB}$, respectively, for 2and $8-\mathrm{MHz}$ bandwidths. For urban RF environments, in which significant low-level interference is present in the GNSS bands, $\sigma_{P}$ can be as high as $0.5 \mathrm{~dB}$ in a $2-\mathrm{MHz}$ bandwidth. This paper's detection test assumes $\sigma_{P}=0.4 \mathrm{~dB}$.

For modeling completeness, the multi-access interference $M(t)$ in (3) can be interpreted in terms of the model for $r_{\mathrm{C}}(t)$ in (7). $M(t)$ models the effect of multi-access interference for a particular desired signal, $r_{\mathrm{A}}(t)$. Invoking the so-called thermal-noise approximation [31], $M(t)$ is assumed to be spectrally flat with density $M_{0}$, whose variable value is a function of the spreading code's chip interval, $\tau_{c}$, and of the average power $\bar{P}_{\mathrm{M}}$ and number $M_{s}$ of multi-access signals. For GNSS signals with a $\operatorname{sinc}^{2}\left(f \tau_{c}\right)$-shaped power spectrum, which applies for all GPS signals except the new military Mcode signals, $M_{0}$ is given by [31]

$$
M_{0}=(2 / 3) M_{s} \bar{P}_{\mathrm{M}} \tau_{c}
$$

Three example cases for $\bar{P}_{\mathrm{M}}$ are given below. In each case, $i=0$ indicates the desired authentic signal and its interferer, 
whereas $i=1, \ldots, M_{s}$ indicate multi-access signals and their interferers.

$\bar{P}_{\mathrm{M}}=P_{\mathrm{A}}$ : In this case, $P_{\mathrm{A} i}=P_{\mathrm{A}}$ for $i=0, \ldots, M_{s}$ and $\eta_{i}=$ 0 for $i=1, \ldots, M_{s}$. In other words, $P_{\mathrm{A}}$ is assumed to be typical of the received power from all GNSS signals and no other interference besides $r_{\mathrm{I} 0}$ is assumed. This case applies for $H_{0}$, for $H_{1}$ when only a single signal, $r_{\mathrm{A} 0}$, experiences significant multipath, and for $\mathrm{H}_{2}$ when the spoofing attack targets only $r_{\mathrm{A} 0}$, as opposed to an attack against all $r_{\mathrm{A} i}$.

$\bar{P}_{\mathrm{M}}=(1+\eta) P_{\mathrm{A}}:$ In this case, $P_{\mathrm{A} i}=P_{\mathrm{A}}$ and $\eta_{i}=\eta$ for $i=$ $0, \ldots, M_{s}$. In other words, each of the $M_{s}+1$ received signals is modeled as having power $P_{\mathrm{A}}$, and each is assumed to be subject to a non-coherent interferer with power $\eta P_{\mathrm{A}}$. This case applies for the jamming hypothesis $H_{3}$, as follows: For all $i,\left|\Delta \tau_{i}\right|$ is assumed to be large enough that $R\left(\Delta \tau_{i}\right)=0$, causing the second term of the summation in (9) to vanish. Jamming power manifests in the first term of the summation as an additional $\eta_{i} P_{\mathrm{A} i}=\eta P_{\mathrm{A}}$.

This case also applies for $\mathrm{H}_{2}$ when the spoofing-to-authentic phase difference $\Delta \theta_{i}$ for $i=0, \ldots, M_{s}$ can be modeled as a random variable uniformly distributed on $[0,2 \pi)$, in which case the second term of the summation in (9) vanishes for all $i$.

$\bar{P}_{\mathrm{M}}=(1+\eta) P_{\mathrm{A}}+2 \sqrt{\eta} P_{\mathrm{A}} \cos (\Delta \theta) R(\Delta \tau)$ : This case applies for $H_{2}$ when the spoofing attack targets not only $r_{\mathrm{A} 0}$ but also the $M_{s}$ multi-access signals such that $\eta_{i} P_{\mathrm{A} i}=\eta P_{\mathrm{A}}$, $\Delta \theta_{i}=\Delta \theta$ and $\Delta \tau_{i}=\Delta \tau$ for $i=0, \ldots, M_{s}$.

\section{B. Symmetric Difference Measurement}

A variety of signal quality monitoring (SQM) metrics have been applied to detect distortions in $\xi_{k}(\tau)$ caused by anomalous GNSS signals, including spoofing signals [21], [22], [40]-[44]. Among these, the so-called symmetric difference is particularly attractive for multipath and spoofing detection because it is simple to implement, is insensitive to the particular shape of the correlation function (provided the function is symmetric about $\tau=0$, as is the case for all current and proposed GNSS signals), and is sensitive to the correlation function distortion introduced by spoofing whenever the authentic and spoofing signals are approximately matched in power (i.e., $0.1<\eta<10$ ). Fig. 5illustrates $\xi_{k}(\tau)$ under nominal conditions (top) and a spoofing attack (bottom).

Let $\tau_{d}$ be the offset of the early and late symmetric difference taps from $\tau=0$, and let $\sigma_{\mathrm{N} 0}$ be the value of $\sigma_{\mathrm{N}}$ in the interference-free case, for which we assume $M_{0}$ is given by 10 with $\bar{P}_{\mathrm{M}}=P_{\mathrm{A}}$. Then at measurement time $t_{k}$ the symmetric difference is calculated as the modulus of the complex difference between the early and late symmetric difference taps, scaled by $1 / \sigma_{\mathrm{NO}}$ :

$$
D_{k}\left(\tau_{d}\right) \triangleq \frac{\left|\xi_{k}\left(-\tau_{d}\right)-\xi_{k}\left(\tau_{d}\right)\right|}{\sigma_{\mathrm{N} 0}}
$$

As with $P_{k}$, a statistical model for $D_{k}\left(\tau_{d}\right)$ is necessary to develop a Bayesian detection framework. Let $p_{D_{k}}(x \mid \theta)$ denote the conditional density of $D_{k}$. An analytical model for $p_{D_{k}}(x \mid \theta)$ is easily derived when there is no coherent interaction between $r_{\mathrm{A}}$ and $r_{\mathrm{I}}$, that is, when the second term of the summation in (9) vanishes, which occurs in the interference-free case $(\eta=0)$ or in the case of jamming, for which $R(\Delta \tau)=0$. Let $P_{H_{0}}$ denote the received power when $\eta=0$, which, as evident in the top panel of Fig. 4 is constant to within a few tenths of a dB. Assume that, for any $r(t)$ and any $k \in\{1,2, \ldots\}$, the AGC adjusts $\beta_{k}$ such that the average power in $\beta_{k} r(t)$ over $t_{k-1}$ to $t_{k}$ is $P_{H_{0}}$. Then, if code tracking is accurate $\left(\Delta \tau_{\mathrm{A} k} \approx 0\right)$, as would be true for up to moderate jamming, or if the amplitude of $\xi_{A}$ is small with respect to $\sigma_{\mathrm{N}}$, as would be true for strong jamming, then the scaled complex difference $\left[\xi_{k}\left(-\tau_{d}\right)-\xi_{k}\left(\tau_{d}\right)\right] / \sigma_{\mathrm{N} 0}$ can be modeled as a zeromean complex Gaussian random variable with variance

$$
\sigma_{d}^{2}=8 \tau_{d}\left(\frac{\beta_{k} \sigma_{\mathrm{N}}}{\sigma_{\mathrm{N} 0}}\right)^{2}
$$

It follows that $p_{D_{k}}(x \mid \theta)$ in this case is Rayleigh with scale parameter $\frac{\sigma_{d}}{\sqrt{2}}$ :

$$
p_{D_{k}}(x \mid \theta)=\frac{2 x}{\sigma_{d}^{2}} \exp \left(\frac{-x^{2}}{\sigma_{d}^{2}}\right), \quad x \geq 0
$$

In the interference-free case, $\beta_{k}=1$ and $\sigma_{\mathrm{N}}=\sigma_{\mathrm{N} 0}$, so $\sigma_{d}^{2}$ reduces to $\sigma_{d}^{2}=8 \tau_{d}$. One can show that this simplified expression for $\sigma_{d}^{2}$ is also approximately true for wideband jamming, for which the AGC maintains $\left(\beta_{k} \sigma_{\mathrm{N}} / \sigma_{\mathrm{N} 0}\right)^{2} \approx 1$. However, the structured-signal jamming assumed in this paper is more potent than wideband jamming: it increases $M_{0}$, and therefore $\sigma_{\mathrm{N}}$, more than equivalently-powered wideband jamming [31]. As a result, $\sigma_{d}^{2}$ rises with increasing power of structured-signal jamming, making it more difficult to distinguish jamming from spoofing. Nevertheless, assuming structured-signal jamming, as opposed to the less potent wideband or narrowband jamming, is consonant with this paper's focus on worst-case attacks.

Note that a version of the symmetric difference normalized by the maximum magnitude of $\xi_{k}(\tau)$ has also been proposed for SQM and spoofing detection [23], [40], [41]. But the definition of $D_{k}$ in 111 is more convenient because its distribution for $\eta=0$ depends only on $\sigma_{d}$, as evident in (13).

Modeling $p_{D_{k}}(x \mid \theta)$ as Rayleigh only holds for $\eta=0$ or $R(\Delta \tau)=0$. When neither of these conditions is true, the real and imaginary components of $\xi_{k}(\tau)$ can manifest strong asymmetry, as shown in the bottom panel of Fig. 5 . In this case, the possible interactions of $\xi_{\mathrm{I} k}(\tau)$, and $\xi_{\mathrm{A} k}(\tau)$ are so complex that $p_{D_{k}}(x \mid \theta)$ cannot be modeled analytically. It can, however, be studied through Monte-Carlo simulation of (5) and (11) as a function of $\theta=[\eta, \Delta \tau, \Delta \theta]^{T}$ and $\tau_{d}$, together with some assumed recipe for determination of $\hat{\tau}$, the receiver's estimate of $\tau_{\mathrm{A}}$. For this paper, we have assumed that

$$
\hat{\tau}=\arg \max _{\tau}\left|\xi_{k}(\tau)\right|
$$

which holds for early-minus-late DLL tracking (both coherent and non-coherent) in the limit as $\tau_{\mathrm{DLL}} \rightarrow 0$, provided the tracking points have not settled on a local maximum different from the global maximum. Note that to minimize the number of correlation taps, one can choose $\tau_{\mathrm{DLL}}=\tau_{d}$, where $\tau_{\mathrm{DLL}}$ is the offset from $\tau=0$ of the DLL's early and late taps. But, depending on the shape of $\xi_{k}(\tau)$ and the assumed distribution 

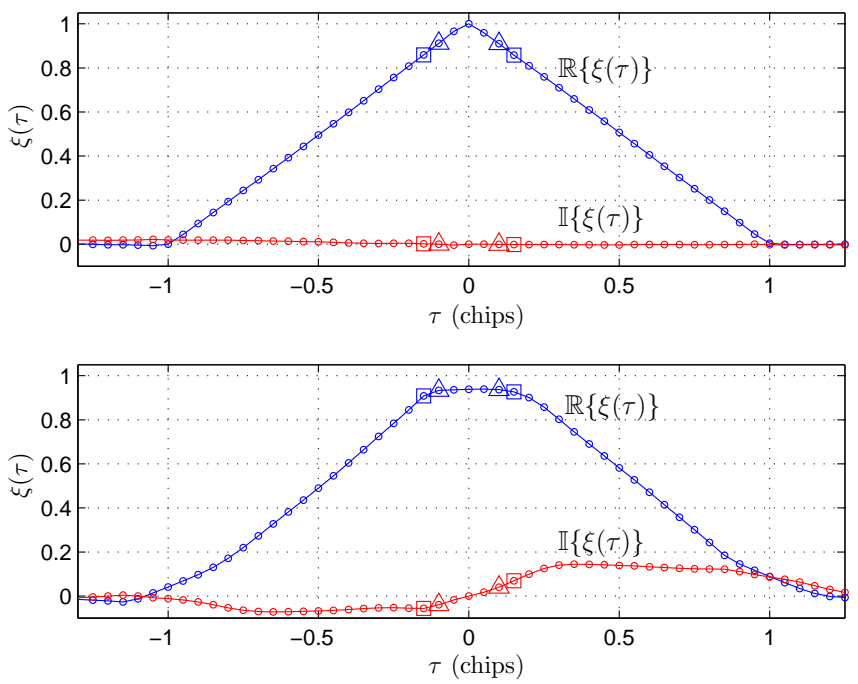

Fig. 5: In-phase $\mathbb{R}\left\{\xi_{k}(\tau)\right\}$ and quadrature $\mathbb{I}\left\{\xi_{k}(\tau)\right\}$ components of the empirical correlation function $\xi_{k}(\tau)$ for a clean scenario (top) and for a spoofing scenario (bottom). The early and late taps used for tracking $\left[\xi_{k}\left(-\tau_{\mathrm{DLL}}\right)\right.$ and $\xi_{k}\left(\tau_{\mathrm{DLL}}\right)$; marked with $\square]$ and for the symmetric difference measurement $\left[\xi_{k}\left(-\tau_{d}\right)\right.$ and $\xi_{k}\left(\tau_{d}\right)$; marked with $\left.\triangle\right]$ are not necessarily the same. The nominal case (top) exhibits symmetry about $\tau=0$ in both components, whereas the spoofed case (bottom) shows distortion and strong asymmetry in both components. Samples of the signal $r(t)$ have been scaled such that $\left|\xi_{k}(0)\right|=1$ for the nominal case.

of $\tau_{\mathrm{I}}$, unequal $\tau_{\mathrm{DLL}}$ and $\tau_{d}$ may improve tracking and detection performance.

In simulation, one can apply whatever algorithm for producing $\hat{\tau}$ best approximates the operation of the receiver, or class of receivers, whose reaction to interference one wishes to simulate. For example, a multipath-mitigating receiver may estimate $\tau_{\mathrm{A}}$ by (1) assuming the presence of one or more multipath components, (2) estimating the parameters $\eta, \tau_{\mathrm{I}}$ and $\theta_{\mathrm{I}}$ for each component, and (3) subtracting a model of each component from $\xi_{k}(\tau)$ [45].

\section{Combined Measurement Vector}

The symmetric difference for tracking channel $i \in$ $\{1,2, \ldots, N\}$ at time $t_{k}$, denoted $D_{k}^{i}\left(\tau_{d}\right)$, can be combined with the received power measurement to form a channelspecific vector of observables at $t_{k}$ :

$$
z_{k}^{i}=\left[D_{k}^{i}\left(\tau_{d}\right), P_{k}\right]^{T}
$$

$D_{k}^{i}\left(\tau_{d}\right)$ and $P_{k}$ are modeled as independent so that

$$
p\left(z_{k}^{i} \mid \theta\right)=p_{D_{k}}\left(z_{1 k}^{i} \mid \theta\right) p_{P_{k}}\left(z_{2 k}^{i} \mid \theta\right)
$$

where $z_{j k}^{i}$ is the $j$ th element of $z_{k}^{i}$.

Obviously, when multiple GNSS signals are simultaneously affected by interference, detection performance improves by considering the multi-channel observation vector

$$
z_{k}^{(1: N)}=\left[D_{k}^{1}\left(\tau_{d}\right), D_{k}^{2}\left(\tau_{d}\right), \ldots, D_{k}^{N}\left(\tau_{d}\right), P_{k}\right]^{T}
$$

For clarity of presentation and analysis, the channel-specific vector $z_{k}^{i}$ has been taken as the observation vector for this paper's Bayesian detection strategy, with the superscript $i$ dropped for notational simplicity. Extension of this paper's methods to the multi-channel case follows straightforwardly from the principles of the per-channel test along with a model for correlation between the $D_{k}^{i}\left(\tau_{d}\right)$.

\section{The Power-Distortion Tradeoff Under Spoofing}

The foregoing measurement models allow one to appreciate the power-distortion tradeoff that a spoofer faces when mounting an attack. In a typical attack, an admixture of spoofing and authentic signals is incident on the receiver's antenna. If the spoofing and authentic signals are approximately matched in power (i.e., $0.1<\eta<10$ ), then the correlation function will be significantly distorted, as shown in the lower panel of Fig. 5. The spoofer has several options for reducing this telltale distortion, but the alternatives are either intrinsically difficult, lead to anomalously high received power, or preclude effective capture, as described below.

\section{A. Nulling or Blocking}

A spoofer can minimize the hallmark distortions of an attack by generating an antipodal, or nulling, signal or by preventing reception of the authentic signal (e.g., by emplacing an obstruction). The form given for $r_{\mathrm{I}}(t)$ in (2) accommodates a nulling attack in which $r_{\mathrm{I}}(t)$ is made antipodal to $r_{\mathrm{A}}(t)$ via the following settings: $\eta=1, \tau_{\mathrm{I}}=\tau_{\mathrm{A}}$, and $\theta_{\mathrm{I}}=\theta_{\mathrm{A}}+\pi$. This attack annihilates $r_{\mathrm{A}}(t)$, producing an effect similar to jamming but with much less received power. But the form in (2) does not accommodate the nulling-and-replacement attack described in [6] and [31], whereby, after nulling $r_{\mathrm{A}}(t)$, the attacker supplants it with a separate spoofing signal. Detection of a perfectly-executed nulling-and-replacement attack is, in fact, not possible with the technique developed in this paper. Such an attack is, however, extremely difficult to carry out in practice, as it requires centimeter-level knowledge of, and a highly accurate fading model for, the attacker-to-receiver signal path.

Similarly, blocking reception of $r_{\mathrm{A}}(t)$ by physical obstruction is difficult in cases where the receiver antenna is not physically accessible to the attacker. The technique developed in this paper makes the assumption that a nulling-and-replacement attack is impractically difficult and that physical access to the receiving antenna is controlled to prevent signal blockage. It must be recognized, however, that in many cases of practical interest (e.g., fishing vessel monitoring), the receiver and antenna are fully under the control of potential attackers.

\section{B. Overpowering}

An alternative approach to eliminating telltale distortion is an overpowered spoofing attack in which $\eta$ is set so high that the receiver's AGC squelches the relatively weak authentic signals below the noise floor, thereby eliminating interaction between $r_{\mathrm{I}}$ and $r_{\mathrm{A}}$. In other words, as $\eta$ increases, $\beta_{k}$ decreases to maintain a constant power in $\beta_{k} r(t)$, with the result that 
$\beta_{k} r_{\mathrm{A}}(t)$ becomes negligible compared to $\beta_{k} r_{\mathrm{I}}(t)$. If, however, the receiver raises an alarm when the received power $P_{k}$ exceeds some threshold, then covert spoofing is strictly limited to $\eta<\eta_{\max }$ for some $\eta_{\max }$.

\section{Underpowering}

The spoofer can also minimize correlation function distortion by selecting a small $\eta$. However, reliable capture of the receiver's tracking loops requires $\eta>\eta_{\min } \approx 0.4 \mathrm{~dB}$ [46]. Thus, for spoofing to be both reliable and covert, $\eta$ is lower bounded by $\eta_{\min }$ and upper bounded by $\eta_{\max }$.

This paper's approach to spoofing detection can be stated as follows: If the defending receiver is designed such that $\eta_{\max }$ is sufficiently low despite maintaining a tolerable rate of false alarm in the received power monitor, then a carry-off spoofing attack that respects this bound yet successfully captures the receiver's tracking loops will unavoidably and detectably distort the correlation function $\xi_{k}(\tau)$. This distortion, evident in $D_{k}\left(\tau_{d}\right)$, can be distinguished from distortion due to multipath by its greater magnitude and by a concomitant increase in $P_{k}$.

\section{Decision Rule}

Finding the decision rule $\delta\left(z_{k}\right)$ from (6) that minimizes the Bayes risk $r(\delta)$ is conceptually straightforward. One can express $r(\delta)$ as

$$
r(\delta)=E\left\{C\left[\delta\left(Z_{k}\right), \Theta\right]\right\}=E\left\{E\left\{C\left[\delta\left(Z_{k}\right), \Theta\right] \mid Z_{k}\right\}\right\}
$$

where the second equality follows from the rule of iterated expectations. We note from (16) that, whatever the distribution of $Z_{k}, r(\delta)$ is minimized when, for each $z_{k} \in \Gamma, \delta$ is chosen to minimize the posterior cost $E\left\{C\left[\delta\left(Z_{k}\right), \Theta\right] \mid Z_{k}=z_{k}\right\}$. Thus, the Bayes-optimal rule is given by

$$
\delta_{\mathrm{B}}\left(z_{k}\right)=\arg \min _{i \in \mathcal{I}} E\left\{C[i, \Theta] \mid Z_{k}=z_{k}\right\}
$$

which, because the parameter sets $\Lambda_{i}$ are disjoint, can be written

$$
\delta_{\mathrm{B}}\left(z_{k}\right)=\arg \min _{i \in \mathcal{I}} \sum_{j=0}^{3} \int_{\Lambda_{j}} C[i, \theta] p\left(\theta \mid z_{k}\right) d \theta
$$

Reversing the conditioning of $p\left(\theta \mid z_{k}\right)$ using Bayes's formula, and recognizing that $w(\theta)=\sum_{j=0}^{3} w_{j}(\theta) \pi_{j}$, we obtain

$$
\delta_{\mathrm{B}}\left(z_{k}\right)=\arg \min _{i \in \mathcal{I}} \sum_{j=0}^{3} \pi_{j} \int_{\Lambda_{j}} C[i, \theta] p\left(z_{k} \mid \theta\right) w_{j}(\theta) d \theta
$$

The foregoing sections defined the parameter sets $\Lambda_{i}$ and presented analytical models for the conditional densities $w_{i}(\theta)$, $i \in \mathcal{I}$. If an analytical model for $p\left(z_{k} \mid \theta\right)$ were also available, then (19) could be solved by numerical integration. Unfortunately, finding an analytical model for $p\left(z_{k} \mid \theta\right)$ only appears possible for the special cases of $\eta=0$ or $R(\Delta \tau)=0$. On the other hand, for a given $\theta$, it is easy to simulate samples drawn from $p\left(z_{k} \mid \theta\right)$ by taking the nonlinear and stochastic models described in Sections [and IV as recipes for a sample simulator. In view of this, a Monte-Carlo technique can be applied to find $\delta_{\mathrm{B}}\left(z_{k}\right)$, as follows.
1) For each $i \in \mathcal{I}, N_{i}$ parameter vectors are simulated according to the distribution $w_{i}(\theta)$, where $N_{i} / N_{\mathrm{P}} \approx \pi_{i}$ and $N_{\mathrm{P}}=\sum_{i \in \mathcal{I}} N_{i}$. The $l$ th simulated vector drawn from $w_{i}(\theta)$ is denoted $\theta_{l i}$.

2) For each $\theta_{l i}, N_{\mathrm{M}}$ simulated measurements $z_{k}$ are generated. Dropping the time index $k$ from $z_{k}$ for notational clarity, the $m$ th simulated measurement, given $\theta_{l i}$, is written $z_{m l i}$. The total number of measurements simulated is $N_{\mathrm{P}} N_{\mathrm{M}}$. Fig. 6 shows an example realization of this sample generation process over a relevant range for $D_{k}$ and $P_{k}$.

3) The two-dimensional observation space $\Gamma$ is divided into a large number of small rectangular cells of uniform size. Each cell is assumed to belong to a single decision region such that all observation samples falling within a cell belonging to $\Gamma_{i}$ are assigned to hypothesis $H_{i}, i \in \mathcal{I}$. An initial partition of $\Gamma$ is created by assigning each cell to the hypothesis $H_{i}$ having the largest number of samples $z_{m l i}$ within the cell. Boundaries are adjusted such that each decision region $\Gamma_{i}$ is simply connected (no islands), a condition that can reasonably be assumed from the fact that $C[i, \theta], p\left(z_{k} \mid \theta\right)$, and $w_{i}(\theta)$ are smooth in $\theta$.

4) The Bayes risk for the current partition is calculated as

$$
r(\delta)=\frac{1}{N_{\mathrm{P}} N_{\mathrm{M}}} \sum_{i=0}^{3} \sum_{l=1}^{N_{i}} \sum_{m=1}^{N_{\mathrm{M}}} C\left[\delta\left(z_{m l i}\right), \theta_{l i}\right]
$$

A new decision region assignment is considered for each cell lying along the boundary between decision regions. The new assignment is retained whenever it reduces $r(\delta)$, provided the resulting decision regions remain simply connected. The process is repeated until no boundary cells warrant re-assigning, at which point the cell assignments constitute the final decision regions $\Gamma_{i}, i \in \mathcal{I}$. Fig. 7 shows an example of the decision regions created by this process.

Two different types of cost function $C[i, \theta]$ are considered, as follows.

\section{A. Uniform Cost within each $\Lambda_{j}$}

When $C[i, \theta]$ is uniform across all $\theta \in \Lambda_{j}$, we write $C_{i j}$, interpreted as the cost of choosing $H_{i}$ when $H_{j}$ is true. The simplest such cost evenly penalizes misclassification: if $i=j$, then $C_{i j}=0$; otherwise, $C_{i j}=1$. But in the context of navigation security, not all types of misclassification are equally costly. We propose the following cost assignment:

$C_{i i}=0, i \in \mathcal{I}$ : Correct decision

$C_{01}=0.2$ : Low cost: Some multipath-induced code- and carrier- phase error might have been mitigated via receiver's multipath mitigation routines had multipath been detected, but otherwise harmless.

$C_{02}=C_{12}=1$ : Highest cost: Spoofing goes undetected; receiver may report hazardously misleading information. Multipath mitigation applied by a receiver deciding $H_{1}$ cannot be assumed to reduce this cost.

$C_{03}=C_{13}=0.9$ : High cost: Jamming goes undetected; receiver may report hazardously misleading information. 


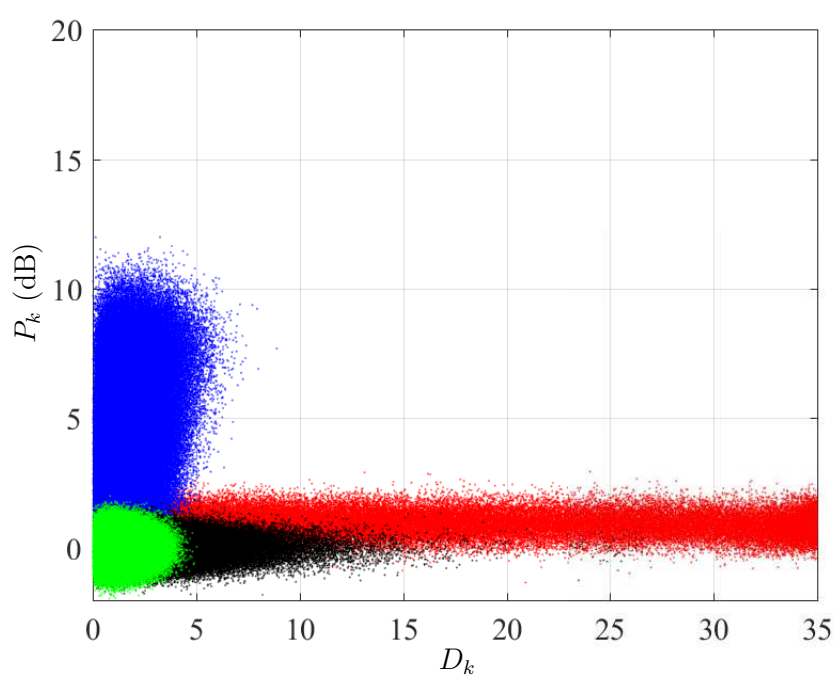

Fig. 6: Simulated observations $z_{k}=\left[D_{k}\left(\tau_{d}\right), P_{k}\right]^{T}$ for no interference (green), multipath (black), spoofing (red), and jamming (blue) based on the Monte-Carlo simulation technique described in the text, with $N_{\mathrm{P}}=10^{5}, N_{\mathrm{M}}=20, \pi_{0}=$ $0.6, \pi_{1}=0.2, \pi_{2}=0.05, \pi_{3}=0.15, \sigma_{P}=0.4 \mathrm{~dB}$, $P_{\mathrm{A}}=-156 \mathrm{dBW}, N_{0}=-204 \mathrm{dBW} / \mathrm{Hz}, M_{s}=7, \tau_{d}=0.15$ chips, $T=100 \mathrm{~ms}, W_{P}=2 \mathrm{MHz}$, and with $\bar{P}_{\mathrm{M}}=P_{\mathrm{A}}$ for $H_{0}$ and $H_{1}, \bar{P}_{\mathrm{M}}=(1+\eta) P_{\mathrm{A}}+2 \sqrt{\eta} P_{\mathrm{A}} \cos (\Delta \theta) R(\Delta \tau)$ for $H_{2}$, and $\bar{P}_{\mathrm{M}}=(1+\eta) P_{\mathrm{A}}$ for $H_{3}$. Note that the spoofing samples are concentrated along a low-power band because the distribution $w_{2}(\theta)$ assumes stealthy spoofing that attempts to masquerade as multipath.

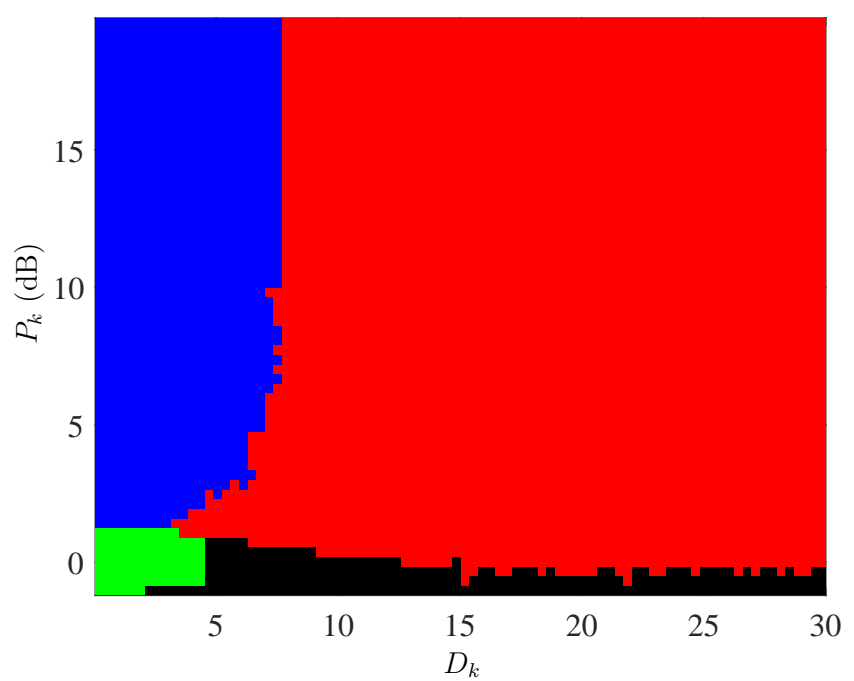

Fig. 7: Optimum decision regions for the $\theta$-dependent cost $C[i, \theta]: \Gamma_{0}$ (no interference, green), $\Gamma_{1}$ (multipath, black), $\Gamma_{2}$ (spoofing, red), and $\Gamma_{3}$ (jamming, blue).

Not so costly as $C_{02}$ because a jammer has less control over receiver output than a spoofer.

$C_{10}=0.1$ : Low cost: Receiver may waste computational resources trying to mitigate phantom multipath, and runs a chance of slightly biasing code and carrier measurements in the process, but otherwise harmless.
$C_{20}=C_{21}=C_{30}=C_{31}=0.4$ : Moderate cost: $\mathrm{A}$ false alarm for jamming or spoofing is raised, breaking navigation solution continuity.

$C_{32}=C_{23}=0.2$ : Low cost: Although spoofing is misclassified as jamming or vice-versa, no hazardously misleading information is issued, as the receiver suppresses its navigation solution when it decides $\mathrm{H}_{2}$ or $\mathrm{H}_{3}$.

\section{B. $\theta$-Dependent Cost}

The harm to a GNSS receiver and its dependent systems or users may not be uniform across all $\theta \in \Lambda_{i}$ for a given $\Lambda_{i} \subset$ $\Lambda, i \in \mathcal{I}$. It is therefore worthwhile to consider the full $\theta$ dependent cost $C[i, \theta]$. Our approach begins with the uniform cost assignment described above, substituting $C[i, \theta], \theta \in \Lambda_{j}$ for certain elements $C_{i j}$, as follows:

$C\left[0, \theta \in \Lambda_{1}\right]=\min \left[0.8, e_{\tau}(\theta) / 0.3\right]$ : Not all multipath is equally harmful: Cost is assumed to increase linearly with the magnitude of multipath-induced code-phase error $e_{\tau}(\theta) \triangleq\left|\hat{\tau}(\theta)-\tau_{A}\right|$, expressed in chips of length $\tau_{c}$, up to a saturation value of 0.8 , where $\hat{\tau}(\theta)$ is given by (14) and $\min [x, y]$ is the minimum of $x$ and $y$.

$C\left[0, \theta \in \Lambda_{2}\right]=C\left[0, \theta \in \Lambda_{1}\right]$ and $C\left[1, \theta \in \Lambda_{2}\right]=0$ if $\Delta \tau<$ $\tau_{\text {DLL }}$ and $\eta<-1 \mathrm{~dB}$; otherwise, $C\left[0, \theta \in \Lambda_{2}\right]=C_{02}$ and $C\left[1, \theta \in \Lambda_{2}\right]=C_{12}$, where $\tau_{\mathrm{DLL}}$ is the offset of the correlation taps used for tracking (marked with $\square$ in Fig. 5): Close-in, weak spoofing is no more harmful than multipath, and can be treated accordingly.

$C\left[0, \theta \in \Lambda_{3}\right]=C\left[1, \theta \in \Lambda_{3}\right]=\min \left[C_{03}, \eta / 10\right]:$ The cost of jamming is assumed to increase linearly with $\eta$ (in $\mathrm{dB}$ ) up to saturation at $C_{03}$.

\section{Prior Probabilities}

The prior probabilities $\pi_{i}, i \in \mathcal{I}$, are of course situationdependent. One may wish to increase $\pi_{2}$, for example, upon entering an area where spoofers have historically been active. Values of $\pi_{i}, i \in \mathcal{I}$ were chosen as follows to represent a heightened threat scenario. Approximate relative values for $\pi_{0}, \pi_{1}$, and $\pi_{3}$ were first found by a manual epoch-by-epoch classification of data from a mobile GPS receiver in an urban environment, which exhibited significant multipath and mild jamming but no spoofing. A low but significant prior probability of spoofing was assumed, and the empirical $\pi_{1}$ and $\pi_{3}$ values were slightly inflated. All values were then normalized such that $\sum_{i \in \mathcal{I}} \pi_{i}=1$. The resulting values, assumed for the remainder of the paper, were $\pi_{0}=0.6, \pi_{1}=0.2, \pi_{2}=0.05$ and $\pi_{3}=0.15$.

\section{Application of the Decision Rule}

The PD detector's Bayes-optimal decision rule $\delta_{\mathrm{B}}$ for classifying received GNSS signals based on observations $z_{k}=\left[D_{k}\left(\tau_{d}\right), P_{k}\right]^{T}$, priors $\pi_{i}$, densities $w_{i}(\theta), p\left(z_{k} \mid \theta\right)$, and the $\theta$-dependent cost $C[i, \theta]$, all as described previously, and with parameter values as indicated in the caption of Fig. 6, is embodied in the colored regions shown in Fig. 7

Application of the decision rule is straightforward: If the observation $z_{k}^{j}$, taken at time $t_{k}$ from tracking channel $j$, falls 
in decision region $\Gamma_{i}$, it is assigned to hypothesis $H_{i}$. Table I shows classification statistics as evaluated by applying the rule to an independent set of Monte-Carlo-generated observations like the one shown in Fig. 6 The table reveals that $\delta_{\mathrm{B}}$ tends to misclassify multipath as $H_{0}$, a consequence of the low cost $C\left[0, \theta \in \Lambda_{1}\right]$, especially when multipath is benign, as is often the case. Multipath is misclassified as spoofing less than $1.7 \%$ of the time even though the modeled $w_{2}(\theta)$ is unfavorable for distinguishing $H_{2}$ from $H_{1}$.

One can achieve a lower spoofing false alarm rate by considering a set of channel-specific decisions over time $\left\{\delta_{\mathrm{B}}\left(z_{k}^{j}\right) \mid k \in\right.$ $\mathcal{K}\}$ for some set $\mathcal{K}$ of contiguous sample times. This approach is effective because a spoofing attack must proceed slowly enough to capture the receiver tracking loops, which offers a window of several seconds over which $\left\{\delta_{\mathrm{B}}\left(z_{k}^{j}\right) \mid k \in \mathcal{K}\right\}$ will include many declarations of $H_{2}$, whereas, under $H_{1}$, only a small number of such declarations would arise. One may also lower the spoofing false alarm rate by considering multi-channel decisions $\left\{\delta_{\mathrm{B}}\left(z_{k}^{j}\right) \mid j=1,2, \ldots, N\right\}$, or the full combined observation in (15). When applying multi-channel tests, one must bear in mind that a spoofer may not attack all channels simultaneously. However, any spoofer wishing to evade simple RAIM-type alarms must produce a selfconsistent signal ensemble [47], which requires spoofing more than $N-N_{m}$ signals, where $N$ is the total number of independent signals being tracked, and $N_{m}$ (typically 4 ) is the minimum number required for a position and time solution.

TABLE I: Simulation-evaluated classification matrix for the decision regions in Fig. 7. The table's $(i, j)$ th element is the relative frequency with which the detector chose $i$ when $j$ was the true scenario.

\begin{tabular}{|c|c|c|c|c|}
\hline \multirow[t]{2}{*}{ Decision } & \multicolumn{4}{|c|}{ True Scenario } \\
\hline & $H_{0}$ & $\overline{H_{1}}$ & $\mathrm{H}_{2}$ & $H_{3}$ \\
\hline$H_{0}$ & 0.9942 & 0.8809 & 0.06244 & 0.0184 \\
\hline$H_{1}$ & 0.0005 & 0.0987 & 0.0234 & 0 \\
\hline $\mathrm{H}_{2}$ & 0.0001 & 0.0162 & 0.8698 & 0.0017 \\
\hline $\mathrm{H}_{3}$ & 0.0039 & 0.0028 & 0.0442 & 0.9799 \\
\hline
\end{tabular}

\section{EXPERIMENTAL DATA}

An independent evaluation of the PD detector was carried out against 27 empirical GNSS data recordings, including 6 recordings of various spoofing scenarios, 14 multipath-dense scenarios, 4 jamming scenarios of different power levels, and 3 scenarios exhibiting negligible interference beyond thermal and multi-access noise. Table $\coprod$ provides a summary description of each recording.

\section{A. TEXBAT}

The Texas Spoofing Test Battery (TEXBAT), version 1.1, from which $\mathrm{cdO}$ and tb2-tb7 are drawn, is a public set of high-fidelity digital recordings of spoofing attacks against civil GPS L1 C/A signals [26]-[28]. Both static and dynamic scenarios are provided along with their corresponding unspoofed recording. Each 16-bit quantized recording is centered at $1575.42 \mathrm{MHz}$ with a bandwidth of $20 \mathrm{MHz}$ and a complex sampling rate of $25 \mathrm{Msps}$. Each spoofing scenario makes use of the most advanced civil GPS spoofer publicly disclosed [3], [48]. In the laboratory, the spoofer can precisely control $\eta, \Delta \tau$, and $\Delta \theta$, and can generate self-consistent and aligned navigation data bits.

Note that tb2-tb 6 exhibit a modest amount of quantization and aliasing noise that makes them easier to detect than would be expected based on this paper's models, whereas tb 7 is free from such extraneous noise. tb 7 is also special in that the spoofer exercises control of $\Delta \theta$, permitting nulling, as described in Section $\mathrm{V}-\mathrm{A}$, in the early stages of the attack. In all other TEXBAT recordings, the spoofer controlled $\eta$ and $\Delta \tau$ but left $\Delta \theta$ at an arbitrary constant value (tb3, tb 4 , and tb6) or allowed it to ramp consistent with the pull-off rate (tb2 and tb5).

\section{B. RNL Multipath and Interference Recordings}

This public set of GNSS recordings, from which wd0-wd12 and sm1-sm3 are drawn, exhibits mild-to-severe multipath and mild unintentional jamming [29], [30]. Static and dynamic scenarios are included in both light and dense urban environments around Austin, TX. Each 16-bit quantized recording is centered at $1575.42 \mathrm{MHz}$ with a bandwidth of $10 \mathrm{MHz}$ and at a complex sampling rate of 12.5 Msps.

\section{Intentional Jamming Recordings}

Jamming scenarios jdl-jd4 were recorded using a personal privacy device generating a sawtooth interference waveform with a sweep range of $1550.02-1606.72 \mathrm{MHz}$ and sweep period of $26 \mu$ s (see [49], Table 1, Row 1 and Fig. 8.). This device typifies low-cost jammers that can be purchased online and easily operated, albeit illegally. The jamming interference was combined with clean, static receiver data from a rooftop antenna and re-recorded. Each 16-bit quantized recording was centered at $1575.42 \mathrm{MHz}$ with a bandwidth of $10 \mathrm{MHz}$ and at a complex sampling rate of $12.5 \mathrm{Msps}$.

\section{Clean Recordings}

Three recordings ( $\mathrm{cdO}$, wdO, and $\mathrm{wd} 1$ ), with negligible interference beyond thermal noise and multi-access interference, were selected from the TEXBAT and RNL Multipath and Interference Recordings data sets. These were all static data sets in quiet RF environments with little multipath.

\section{E. Pre-Processing}

Raw wideband complex samples were first processed by the GRID science-grade software-defined receiver [50] to produce $100-\mathrm{Hz}$ complex accumulations free of navigation data modulation at 41 uniformly-spaced taps spanning the range $\left[-\tau_{c}, \tau_{c}\right]$ around the prompt tap $(\tau=0)$. From the resulting data-free $100-\mathrm{Hz}$ accumulations, the nominal thermal noise deviation $\sigma_{\mathrm{N} 0}$ needed to form $D_{k}$ in (11) was estimated by taking a complex-accumulation-wise standard deviation across multiple channels and multiple correlation tap offsets over a 15 second period in each recording that was substantially free from interference. Blocks of $10100-\mathrm{Hz}$ 
TABLE II: Summary of 27 data recordings used for experimental evaluation.

\begin{tabular}{llll}
\hline Type & ID & Description & Duration (s) \\
\hline$H_{0}$ & cd0 & static rooftop & 456 \\
$H_{0}$ & wd0 & static open field & 304 \\
$H_{0}$ & wd1 & static open field & 298 \\
$H_{1}$ & wd2 & dynamic deep urban & 298 \\
$H_{1}$ & wd3 & dynamic deep urban & 456 \\
$H_{1}$ & wd4 & dynamic deep urban & 292 \\
$H_{1}$ & wd5 & dynamic deep urban & 292 \\
$H_{1}$ & wd6 & dynamic deep urban & 460 \\
$H_{1}$ & wd7 & dynamic deep urban & 246 \\
$H_{1}$ & wd8 & dynamic deep urban & 278 \\
$H_{1}$ & wd9 & dynamic deep urban & 334 \\
$H_{1}$ & wd10 & dynamic deep urban & 370 \\
$H_{1}$ & wd11 & dynamic deep urban & 390 \\
$H_{1}$ & wd12 & dynamic deep urban & 390 \\
$H_{1}$ & sm1 & static urban & 1700 \\
$H_{1}$ & sm2 & static urban & 600 \\
$H_{1}$ & sm3 & static urban & 150 \\
$H_{2}$ & tb2 & static time push, $\eta=10 \mathrm{~dB}$ & 346 \\
$H_{2}$ & tb3 & static time push, $\eta=1.3 \mathrm{~dB}$ & 336 \\
$H_{2}$ & tb4 & static pos. push, $\eta=0.4 \mathrm{~dB}$ & 336 \\
$H_{2}$ & tb5 & dynamic pos. push, $\eta=9.9 \mathrm{~dB}$ & 304 \\
$H_{2}$ & tb6 & static time push, $\eta=0.8 \mathrm{~dB}$ & 308 \\
$H_{2}$ & tb7 & static time push, $\Delta \theta$ control & 468 \\
$H_{3}$ & jd1 & PPD, $\eta=18 \mathrm{~dB}$ & 108 \\
$H_{3}$ & jd2 & PPD, $\eta=7 \mathrm{~dB}$ & 58 \\
$H_{3}$ & jd3 & PPD, $\eta=8 \mathrm{~dB}$ & 108 \\
$H_{3}$ & jd4 & PPD, $\eta=2 \mathrm{~dB}$ & 108 \\
\hline & & &
\end{tabular}

complex accumulations were then averaged to create $10-\mathrm{Hz}$ accumulations, at each of the 41 code phase offsets. These $10-\mathrm{Hz}$ complex accumulations are those modeled by $\xi_{k}(\tau)$ in Fig. 1.

Received power was calculated directly from the raw wideband (e.g., $25 \mathrm{Msps}$ ) complex samples by filtering to a bandwidth $W_{P}$ of $2 \mathrm{MHz}$, as in Fig. 3, then averaging the squared modulus of the filtered samples over $200 \mathrm{~ms}$ to produce a 5$\mathrm{Hz}$ time history of received power. This was aligned in time with the $\xi_{k}(\tau)$ and interpolated to produce a corresponding 10-Hz $P_{k}$.

Naturally-occurring low-level (less than $2 \mathrm{~dB}$ ) unintentional jamming was present in many of the type- $H_{1}$ (multipath) data recordings taken in urban settings. Fig. 8 shows an example $P_{k}$ time history exhibiting such jamming as intermittent spikes. The short data intervals containing these spikes were excised from these recordings to ensure they exhibited only multipath and thermal noise. Otherwise, the PD detector would classify the type- $H_{1}$ recordings as a mixture of multipath and jamming, which, although true, would complicate an analysis of misclassification using the empirical data. As shown in the bottom panel of Fig. 8, the cumulative distribution of power for each data set was used to identify the distribution tail, at whose boundary the excision threshold was set.

\section{EXPERIMENTAL RESULTS}

This section documents the experimental performance assessment of the PD detector, when applying the decision regions shown in Fig. 7, against the 27 recordings listed in Table III Because tb7 is a special case that violates the PD detector's assumption against nulling, it will be treated separately in the discussion below.
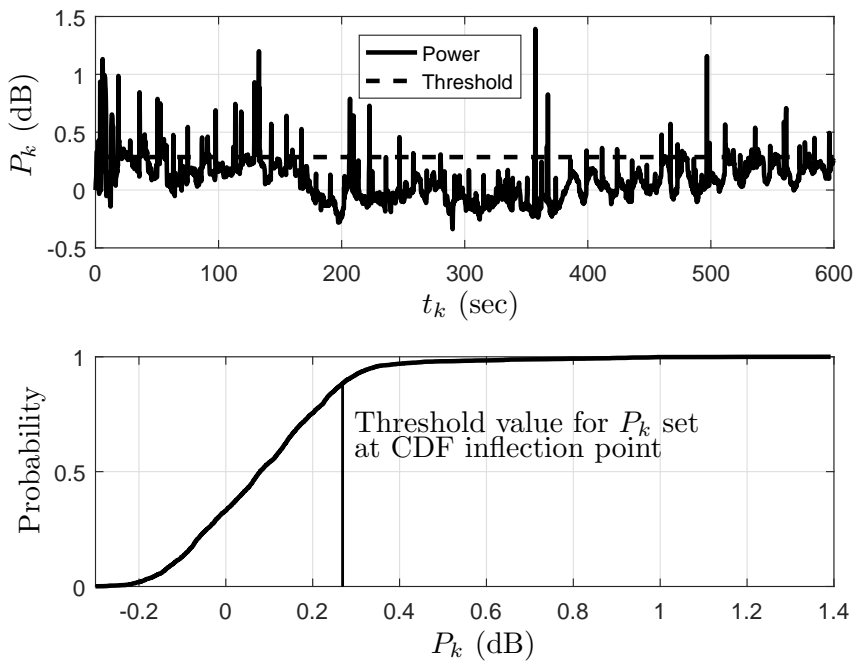

Fig. 8: Top: Time history of received power measurements $P_{k}$ for data set sm2. Power spikes indicate the presence of low-level unintentional jamming. Bottom: The inflection point of the empirical cumulative distribution function (CDF) was taken as the threshold above which the empirical data were deemed to be of type $H_{3}$ (jamming) instead of type $H_{1}$ (multipath), and so excised from the recording.

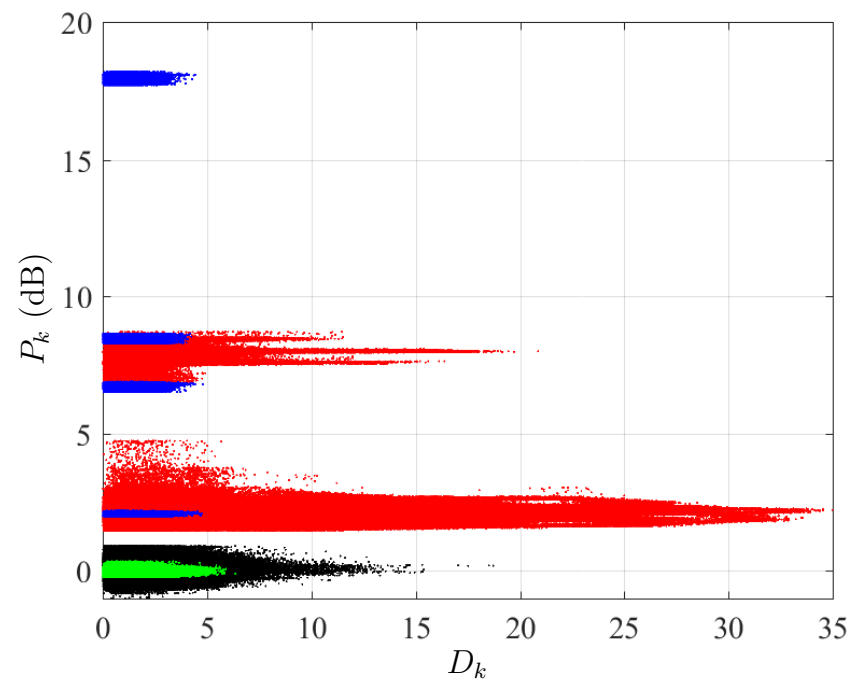

Fig. 9: Observations $z_{k}=\left[D_{k}, P_{k}\right]^{T}$ for clean (green), multipath (black), spoofing (red), and jamming (blue) from the experimental recordings.

Observations $z_{k}$ from the experimental recordings (sans tb 7) are shown in Fig. 9. Clearly, the simulated observations in Fig. 6 agree well with the empirical ones. The abrupt upper boundary of the multipath samples is due to the thresholding discussed in connection with Fig. 8

Fig. 10 shows a single-channel cumulative time history of the PD detector's decisions for example jamming (top panel) and spoofing (bottom panel) attack scenarios. In the jamming scenario, the attack is detected immediately at onset, and continuously declared so thereafter. In the spoofing scenario, the attack is detected immediately, but initially classified as 

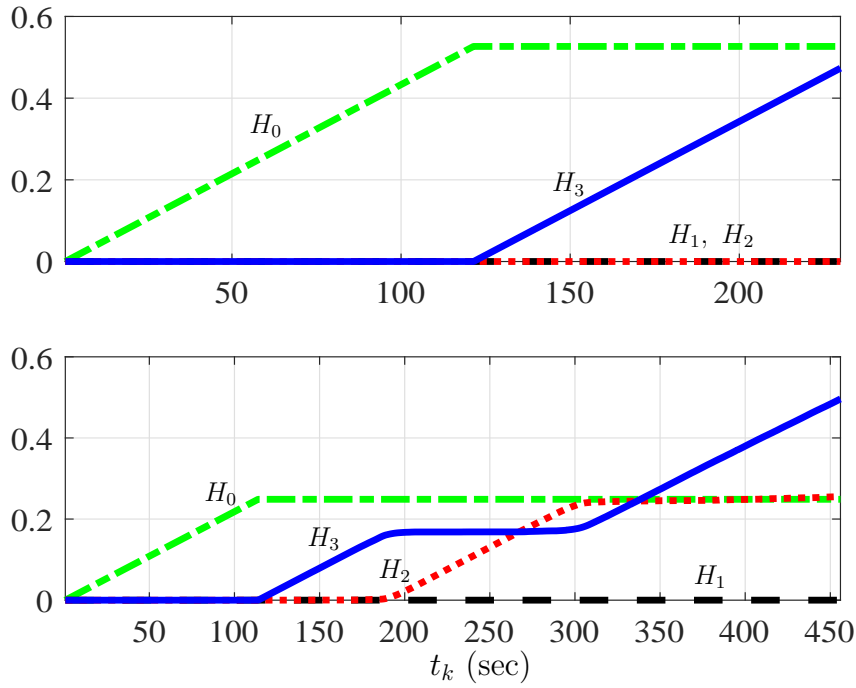

Fig. 10: Cumulative time history of decisions $\delta_{\mathrm{B}}\left(z_{k}\right)$ for a single receiver tracking channel. Each trace represents the total number of times the corresponding hypothesis was chosen up to time $t_{k}$, normalized so that the final cumulative values sum to one. Both attacks begin at 120 seconds. Top: Jamming scenario jd3. Bottom: Spoofing scenario tb 4 with the detector applied to a mid-elevation satellite signal.

jamming because the spoofer's near-perfect initial code-phase alignment $(\Delta \tau \approx 0)$ causes little distortion (and, indeed, little harm to the receiver). At about 180 seconds, the spoofer begins its pull-off, whereupon the increased correlation function distortion reveals the attack as spoofing. After $t_{k}=300$, the pull-off has proceeded far enough that correlation function distortion is less pronounced and the attack is declared to be jamming again. It is notable that the spoofing attack is caught despite its low power advantage, which was $\eta=0.4 \mathrm{~dB}$ as intended, but $\sim 1.5 \mathrm{~dB}$ in effect due to quantization noise in the spoofer.

Table III summarizes the PD detector's overall performance against the experimental data in terms of classification statistics. Importantly, all instances of spoofing and jamming were flagged as attacks. However, whereas jamming was always categorized correctly, spoofing was declared to be jamming $>4 / 5$ of the time, much higher than the simulated-data spoofingto-jamming misclassification rate presented in Table I] The difference is that the experimental attacks all begin initially code-phase aligned $(\Delta \tau \approx 0)$ and so do not initially cause harm or significant distortion. Moreover, as pull-off proceeds and $\Delta \theta$ exceeds $\tau_{c}$, distortion subsides, becoming negligible beyond $2 \tau_{c}$, whereupon the spoofing is either classified as $H_{0}$ (if low-power) or $H_{3}$ (if high-power). Thus, the PD detector is most effective at recognizing spoofing as such during initial carry-off of the tracking points. Conveniently, this is precisely when (1) carry-off-type spoofing begins to be hazardous, and when (2) civil GNSS spoofing detection strategies based on cryptographic security codes, as in [8], are least effective. Thus, the PD detector is nicely complementary with the methods of [8].

A second important result in Table III is the low rate of false spoofing or jamming alarms. Clean $\left(H_{0}\right)$ data produced no false alarms, and multipath-rich data $\left(H_{1}\right)$ produced false alarms only $0.57 \%$ of the time. Thus, for a multi-channel decision, assuming an urban setting with independent multipath across tracking channels and $t_{k+1}-t_{k}=100 \mathrm{~ms}$, any subset of 6 from a total of $N \leq 20$ tracking channels would only simultaneously false alarm on average once every 2.5 years.

TABLE III: As Table \but for the PD detector applied to the experimental recordings (sans tb 7 ).

\begin{tabular}{cccccc}
\hline Decision & & \multicolumn{4}{c}{ True Scenario } \\
\cline { 3 - 6 } \cline { 3 - 5 } & $H_{0}$ & $H_{1}$ & $H_{2}$ & $H_{3}$ \\
\hline$H_{0}$ & & 1 & 0.8717 & 0 & 0 \\
$H_{1}$ & & 0 & 0.1226 & 0 & 0 \\
$H_{2}$ & & 0 & 0.0057 & 0.1784 & 0 \\
$H_{3}$ & 0 & 0 & 0.8217 & 1 \\
\hline
\end{tabular}

To test its limits, the PD detector was applied to tb 7 , an especially subtle attack in which the spoofer carefully controls $\Delta \theta$, effects authentic signal nulling during the initial stages of the attack, and maintains an approximately constant measured signal amplitude during pull-off [28]. As explained in Section $\mathrm{V}-\mathrm{A}$, such an attack would be difficult to mount outside the laboratory. As might be expected, the detector's performance was worse for this attack than for the other spoofing attacks: its decision rates during the attack portion of the recording were $H_{0}: 14 \%, H_{1}: 10 \%, H_{2}: 70 \%$, and $H_{3}: 6 \%$. Nonetheless, the attack was caught on each channel soon after pull-off began.

\section{CONClusions}

We presented the PD detector, a novel low-cost, receiverautonomous, readily-implementable GNSS jamming and carry-off spoofing detector. The detector traps a would-be attacker between simultaneous monitoring of received power and complex correlation function distortion. It amounts to a multihypothesis Bayesian classifier applied to a problem with three unknown parameters whose prior distributions are informed by the physics of GNSS signal reception and signal processing, and whose prior probabilities can be adjusted to reflect the threat environment in which a receiver operates. In evaluation against 27 high-quality experimental recordings of attack and non-attack scenarios, the detector correctly alarmed on all malicious attacks while maintaining a single-channel false alarm rate below $0.6 \%$. For convenient implementation, the PD detector's decision rule for three different cost functions, together with all code required to generate application-tailored decision rules, is available at https://github.com/navSecurity/PD-defense.

\section{ACKNOWLEDGEMENTS}

Jason Gross's work on this project was supported in part by a West Virginia University Big XII Faculty Fellowship. Todd Humphreys's work on this project has been supported by the National Science Foundation under Grant No. 1454474 (CAREER) and by the Data-supported Transportation Operations and Planning Center (DSTOP), a Tier 1 USDOT University Transportation Center. 


\section{REFERENCES}

[1] GPS Directorate, "Systems engineering and integration Interface Specification IS-GPS-200G," 2012, http://www.gps.gov/technical/icwg/

[2] European Union, "European GNSS (Galileo) open service signal in space interface control document," 2010, http://ec.europa.eu/enterprise/policies/satnav/galileo/open-service/

[3] T. E. Humphreys, B. M. Ledvina, M. L. Psiaki, B. W. O'Hanlon, and P. M. Kintner, Jr., "Assessing the spoofing threat: Development of a portable GPS civilian spoofer," in Proceedings of the ION GNSS Meeting. Savannah, GA: Institute of Navigation, 2008.

[4] John A. Volpe National Transportation Systems Center, "Vulnerability assessment of the transportation infrastructure relying on the Global Positioning System," 2001.

[5] D. P. Shepard, T. E. Humphreys, and A. A. Fansler, "Evaluation of the vulnerability of phasor measurement units to GPS spoofing attacks," International Journal of Critical Infrastructure Protection, vol. 5, no. 3-4, pp. 146-153, 2012

[6] M. L. Psiaki and T. E. Humphreys, "GNSS spoofing and detection," Proceedings of the IEEE, vol. 104, no. 6, pp. 1258-1270, 2016.

[7] K. D. Wesson, M. P. Rothlisberger, and T. E. Humphreys, "Practical cryptographic civil GPS signal authentication," Navigation, Journal of the Institute of Navigation, vol. 59, no. 3, pp. 177-193, 2012.

[8] T. E. Humphreys, "Detection strategy for cryptographic GNSS antispoofing," IEEE Transactions on Aerospace and Electronic Systems, vol. 49, no. 2, pp. 1073-1090, 2013.

[9] A. J. Kerns, K. D. Wesson, and T. E. Humphreys, "A blueprint for civil GPS navigation message authentication," in Proceedings of the IEEE/ION PLANS Meeting, May 2014.

[10] I. Fernández-Hernández, V. Rijmen, G. Seco-Granados, J. Simon, I. Rodríguez, and J. D. Calle, "A navigation message authentication proposal for the Galileo open service," Navigation, vol. 63, no. 1, pp. 85-102, 2016.

[11] M. Psiaki, B. O'Hanlon, J. Bhatti, D. Shepard, and T. Humphreys, "GPS spoofing detection via dual-receiver correlation of military signals," IEEE Transactions on Aerospace and Electronic Systems, vol. 49, no. 4, pp. 2250-2267, 2013.

[12] P. Y. Montgomergy, T. E. Humphreys, and B. M. Ledvina, "Receiverautonomous spoofing detection: Experimental results of a multi-antenna receiver defense against a portable civil GPS spoofer," in Proceedings of the ION International Technical Meeting, Anaheim, CA, Jan. 2009.

[13] M. L. Psiaki, B. W. O'Hanlon, S. P. Powell, J. A. Bhatti, K. D. Wesson, and T. E. Humphreys, "GNSS spoofing detection using two-antenna differential carrier phase," in Proceedings of the 27th Meeting of the Satellite Division of the Institute of Navigation (ION GNSS+ 2014). ION, 2014.

[14] M. Meurer, A. Konovaltsev, M. Cuntz, and C. Hättich, "Robust joint multi-antenna spoofing detection and attitude estimation using direction assisted multiple hypotheses RAIM," in Proceedings of the 25th Meeting of the Satellite Division of the Institute of Navigation (ION GNSS+ 2012). ION, 2012.

[15] D. Borio, "PANOVA tests and their application to GNSS spoofing detection," IEEE Transactions on Aerospace and Electronic Systems, vol. 49, no. 1, pp. 381-394, Jan. 2013.

[16] M. Psiaki, S. P. Powell, and B. W. O'Hanlon, "GNSS spoofing detection using high-frequency antenna motion and carrier-phase data," in Proceedings of the ION GNSS+ Meeting, 2013, pp. 2949-2991.

[17] E. McMilin, D. S. De Lorenzo, T. Walter, T. H. Lee, and P. Enge, "Single antenna GPS spoof detection that is simple, static, instantaneous and backwards compatible for aerial applications," in Proceedings of the 27th International Technical Meeting of The Satellite Division of the Institute of Navigation (ION GNSS+ 2014), Tampa, FL, 2014, pp. 2233-2242.

[18] N. White, P. Maybeck, and S. DeVilbiss, "Detection of interference/jamming and spoofing in a DGPS-aided inertial system," IEEE Transactions on Aerospace and Electronic Systems, vol. 34, no. 4, pp. $1208-1217,1998$

[19] C. Tanıl, S. Khanafseh, and B. Pervan, "GNSS spoofing attack detection using aircraft autopilot response to deceptive trajectory," in Proceedings of the ION GNSS+ Meeting. ION, 2015.

[20] D. M. Akos, "Who's afraid of the spoofer? GPS/GNSS spoofing detection via automatic gain control (AGC)," Navigation, Journal of the Institute of Navigation, vol. 59, no. 4, pp. 281-290, 2012.

[21] B. M. Ledvina, W. J. Bencze, B. Galusha, and I. Miller, "An in-line anti-spoofing module for legacy civil GPS receivers," in Proceedings of the ION International Technical Meeting, San Diego, CA, Jan. 2010.
[22] A. Cavaleri, B. Motella, M. Pini, and M. Fantino, "Detection of spoofed GPS signals at code and carrier tracking level," in 5th ESA Workshop on Satellite Navigation Technologies and European Workshop on GNSS Signals and Signal Processing, Dec. 2010.

[23] J. Huang, L. L. Presti, B. Motella, and M. Pini, "GNSS spoofing detection: Theoretical analysis and performance of the ratio test metric in open sky," ICT Express, vol. 2, no. 1, pp. 37-40, 2016.

[24] R. Mitch, R. Dougherty, M. Psiaki, S. Powell, B. O'Hanlon, J. Bhatti, and T. E. Humphreys, "Know your enemy: Signal characteristics of civil GPS jammers," GPS World, Jan. 2012.

[25] K. D. Wesson, B. L. Evans, and T. E. Humphreys, "A combined symmetric difference and power monitoring GNSS anti-spoofing technique," in IEEE Global Conference on Signal and Information Processing, 2013.

[26] T. E. Humphreys, J. A. Bhatti, D. P. Shepard, and K. D. Wesson, "The Texas Spoofing Test Battery: Toward a standard for evaluating GNSS signal authentication techniques," in Proceedings of the ION GNSS Meeting, 2012, http://radionavlab.ae.utexas.edu/texbat

[27] T. R. Laboratory, "Texas Spoofing Test Battery (TEXBAT)," July 2017, http://radionavlab.ae.utexas.edu/texbat

[28] T. Humphreys, "TEXBAT data sets 7 and 8," 2016, http://radionavlab.ae.utexas.edu/datastore/texbat/texbat_ds7_and_ds8.pdf

[29] K. D. Wesson, D. P. Shepard, J. A. Bhatti, and T. E. Humphreys, "An evaluation of the vestigial signal defense for civil GPS anti-spoofing," in Proceedings of the ION GNSS Meeting, Portland, OR, 2011.

[30] K. Pesyna, Z. Kassas, J. Bhatti, and T. Humphreys, "Tightly-coupled opportunistic navigation for deep urban and indoor positioning," in Proceedings of the International Technical Meeting of The Satellite Division of the Institute of Navigation (ION GNSS), vol. 1, 2011, pp. 3605-3617.

[31] T. E. Humphreys, Springer Handbook of Global Navigation Satellite Systems. Springer, 2017, ch. Interference, pp. 169-504.

[32] R. D. J. Van Nee, "Spread-spectrum code and carrier synchronization errors caused by multipath and interference," IEEE Transactions on Aerospace and Electronic Systems, vol. 29, no. 4, pp. 1359-1365, 1993.

[33] A. J. Van Dierendonck, P. Fenton, and T. Ford, "Theory and performance of narrow correlator spacing in a GPS receiver," Navigation, Journal of the Institute of Navigation, vol. 39, no. 3, pp. 265-283, Fall 1992.

[34] H. V. Poor, An Introduction to Signal Detection and Estimation, 2nd Edition. Springer, 1994.

[35] D. Hume and T. L. Beauchamp, An enquiry concerning human understanding: A critical edition. Oxford University Press, 2000, vol. 3.

[36] G. L. Turin, F. D. Clapp, T. L. Johnston, S. B. Fine, and D. Lavry, "A statistical model of urban multipath propagation," IEEE Transactions on Vehicular Technology, vol. VT-21, no. 1, Feb. 1972.

[37] E. Steingass and A. L. German, "Measuring the navigation multipath channel-a statistical analysis," in Proceedings of the ION GNSS Meeting, 2004.

[38] A. Lehner, Multipath Channel Modelling for Satellite Navigation Systems. Shaker, 2007, ISBN: 978-3-8322-6651-6.

[39] P. W. Ward, "GPS receiver RF interference monitoring, mitigation, and analysis techniques," Navigation, Journal of the Institute of Navigation, vol. 41, no. 4, pp. 367-391, 1994.

[40] R. E. Phelts, Multicorrelator Techniques for Robust Mitigation of Threats to GPS Signal Quality. Stanford University, 2001.

[41] M. Irsigler and G. Hein, "Development of a real time multipath monitor based on multi-correlator observations," in Proceedings of the ION GNSS Meeting, 2005.

[42] S. Gunawardena, Z. Zhu, M. U. de Haag, and F. van Graas, "Remotecontrolled, continuously operating GPS anomalous event monitor," Navigation, Journal of the Institute of Navigation, vol. 56, no. 2, pp. 97-113, 2009.

[43] O. M. Mubarak and A. G. Dempster, "Analysis of early late phase in single- and dual frequency GPS receivers for multipath detection," GPS Solut, vol. 14, pp. 381-388, Feb. 2010.

[44] M. T. Gamba, M. D. Truong, B. Motella, E. Falletti, and T. H. Ta, "Hypothesis testing methods to detect spoofing attacks: a test against the TEXBAT datasets," GPS Solutions, pp. 1-13, 2016.

[45] L. R. Weill, "Multipath mitigation using modernized GPS signals: How good can it get?" in Proceedings of the ION GPS Meeting, Portland, OR, Sept. 2002.

[46] D. Shepard and T. E. Humphreys, "Characterization of receiver response to a spoofing attack," in Proceedings of the ION GNSS Meeting. Portland, Oregon: Institute of Navigation, 2011.

[47] S. Khanafseh, N. Roshan, S. Langel, F.-C. Chan, M. Joerger, and B. Pervan, "GPS spoofing detection using RAIM with INS coupling," in Position, Location and Navigation Symposium-PLANS 2014, 2014 IEEE/ION. IEEE, 2014, pp. 1232-1239. 
[48] T. E. Humphreys, D. P. Shepard, J. A. Bhatti, and K. D. Wesson, "A testbed for developing and evaluating GNSS signal authentication techniques," in Proceedings of the International Symposium on Certification of GNSS Systems and Services (CERGAL), Dresden, Germany, July 2014.

[49] R. Mitch, R. Dougherty, M. Psiaki, S. Powell, B. O'Hanlon, J. Bhatti, and T. Humphreys, "Signal characteristics of civil GPS jammers," in Proceedings of the ION GNSS Meeting, 2011.

[50] E. G. Lightsey, T. E. Humphreys, J. A. Bhatti, A. J. Joplin, B. W. O'Hanlon, and S. P. Powell, "Demonstration of a space capable miniature dual frequency GNSS receiver," Navigation, Journal of the Institute of Navigation, vol. 61, no. 1, pp. 53-64, 2014. 